\title{
Hippocampal Place Cells Can Encode Multiple Trial-Dependent Features through Rate Remapping
}

\author{
Kevin Allen, ${ }^{1,2}$ J. Nick P. Rawlins, ${ }^{3}$ David M. Bannerman, ${ }^{3}$ and Jozsef Csicsvari ${ }^{1,4}$ \\ ${ }^{1}$ MRC Anatomical Neuropharmacology Unit, Department of Pharmacology, University of Oxford, Oxford, OX1 3TH, United Kingdom, ${ }^{2}$ Department of \\ Clinical Neurobiology, Heidelberg University Hospital and German Cancer Research Center (DKFZ), INF 280, 69120 Heidelberg, Germany, ${ }^{3}$ Department of \\ Experimental Psychology, University of Oxford, Oxford, OX1 3UD, United Kingdom, and ${ }^{4}$ Institute of Science and Technology Austria, Klosterneuburg, \\ A-3400, Austria
}

The activity of hippocampal pyramidal cells reflects both the current position of the animal and information related to its current behavior. Here we investigated whether single hippocampal neurons can encode several independent features defining trials during a memory task. We also tested whether task-related information is represented by partial remapping of the place cell population or, instead, via firing rate modulation of spatially stable place cells. To address these two questions, the activity of hippocampal neurons was recorded in rats performing a conditional discrimination task on a modified T-maze in which the identity of a food reward guided behavior. When the rat was on the central arm of the maze, the firing rate of pyramidal cells changed depending on two independent factors: (1) the identity of the food reward given to the animal and (2) the previous location of the animal on the maze. Importantly, some pyramidal cells encoded information relative to both factors. This trial-type specific and retrospective coding did not interfere with the spatial representation of the maze: hippocampal cells had stable place fields and their theta-phase precession profiles were unaltered during the task, indicating that trial-related information was encoded via rate remapping. During error trials, encoding of both trialrelated information and spatial location was impaired. Finally, we found that pyramidal cells also encode trial-related information via rate remapping during the continuous version of the rewarded alternation task without delays. These results suggest that hippocampal neurons can encode several task-related cognitive aspects via rate remapping.

\section{Introduction}

Hippocampal pyramidal cells fire in relation to space (O'Keefe and Dostrovsky, 1971). In addition, the firing of hippocampal cells can be altered by contextual cues, such as the color of the walls during open-field exploration (Jeffery and Anderson, 2003; Leutgeb et al., 2005) or task-contingent information during spatial tasks (Frank et al., 2000; Wood et al., 2000; Ferbinteanu and Shapiro, 2003; Bower et al., 2005; Lee et al., 2006; Shapiro and Ferbinteanu, 2006; Ainge et al., 2007a,b; Pastalkova et al., 2008).

During spatial tasks, hippocampal neurons often fire in a trialdependent manner. For example, in spatial working memory tasks the firing of some hippocampal cells is controlled by the previous or future location of the animal (Frank et al., 2000; Wood et al., 2000). This suggests that these firing changes represent retrospective or prospective memory traces incorporating

Received Dec. 12, 2011; revised July 31, 2012; accepted Aug. 25, 2012.

Author contributions: K.A., J.N.P.R., D.M.B., and J.C. designed research; K.A. performed research; K.A. and J.C. analyzed data; K.A., J.N.P.R., D.M.B., and J.C. wrote the paper.

J.C. was supported by a MRC Intramural Programme Grant (U138197111) and a European Research Council Starter Grant (281511). K.A. held a Wellcome Trust PhD studentship and a Humboldt Research Fellowship for Postdoctoral Researchers. D.M.B. was supported by Wellcome Trust Senior Fellowships (074385 and 087736 ). We thank D. Dupret, J. O'Neill, and B. Pleydell-Bouverie for their constructive comments on this manuscript, 0 . Potvin for his help with the recordings, and N. Campo-Urriza for the technical assistance.

Correspondence should be addressed to Dr. Jozsef Csicsvari, Institute of Science and Technology Austria, Klosterneuburg, Am Campus 1, A-3400, Austria. E-mail: jozsef.csicsvari@ist.ac.at.

DOI:10.1523/JNEUROSCI.6175-11.2012

Copyright $\odot 2012$ the authors $\quad 0270-6474 / 12 / 3214752-15 \$ 15.00 / 0$ the past experience of the animal and its future choice. It is also possible that during a memory task the firing rate of pyramidal cells changes on single trials to encode not only single features, but also combinations of multiple features associated with both past and future locations. This has not been examined directly.

Some studies have suggested that prospective and retrospective coding cells might be active only during some trial-types or that the location of their firing fields changes across trials of different types (Wood et al., 2000; Ferbinteanu and Shapiro, 2003; Lee et al., 2006; Kennedy and Shapiro, 2009), indicating that the hippocampal spatial representation undergoes global or partial remapping. However, an alternative explanation is possible; contextual information could be expressed by rate remapping, which involves firing rate changes of place cells without alterations of their spatial selectivity (Leutgeb et al., 2005). Such rate remapping has been suggested to take place during certain memory tasks as well (Lipton et al., 2007; Ferbinteanu et al., 2011; Ainge et al., 2012), although this has not been quantitatively tested. Rate remapping would enable simultaneous coding of place and additional features. Indeed, hippocampal neurons could encode spatial information by the timing of their action potentials relative to ongoing theta oscillations (O'Keefe and Recce, 1993) and the instantaneous firing rate could encode trial-related information (Huxter et al., 2003). Resolving the nature of remapping during memory tasks would shed light on the mechanisms responsible for task-contingent firing.

In this study, the activity of hippocampal neurons was recorded in a task in which non-spatial stimuli guided the animal's 
A

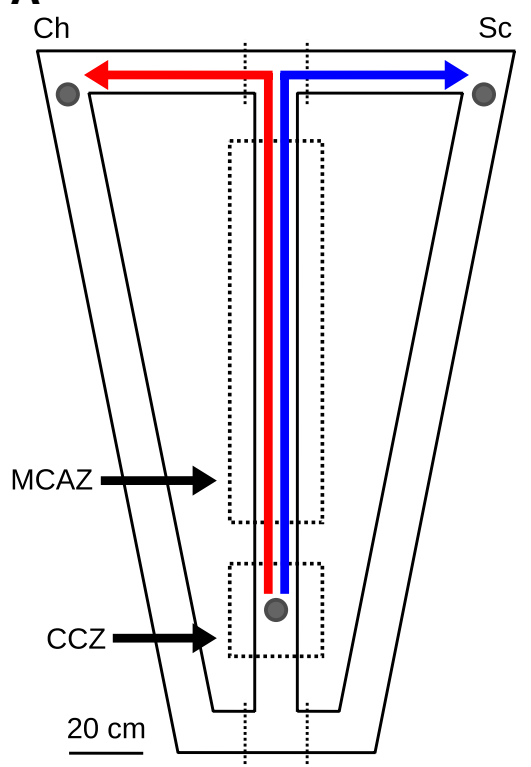

B

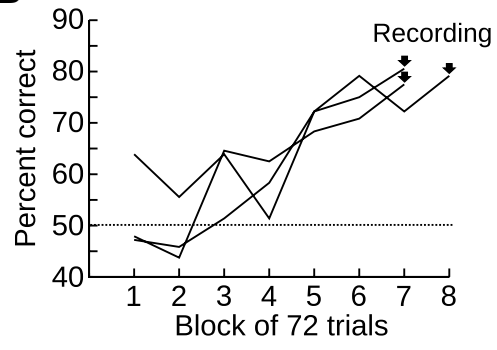

C

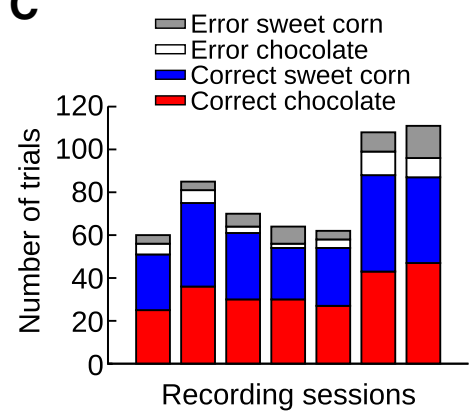

Figure 1. Food-location conditional discrimination task. $A$, Schematic of the modified T-maze. The location of each inverted guillotine door used in the experiment is indicated by a dotted line. Gray circles represent the food wells. The two dotted rectangles correspond to the zones used for the analysis at the food well of the central arm (CCZ) and on the middle portion of the central arm (MCAZ). The two colored arrows indicate the correct response to perform for a rat on trials in which chocolate (Ch; red arrow) or sweet corn (Sc; blue arrow) was given as a reward on the central arm of the maze. $\boldsymbol{B}$, Acquisition of the conditional discrimination task by three rats. Chance level is indicated by a dotted line. The recording began at the end of each learning curve, as indicated by the arrows. C, Number of trials and performance during the recording sessions used for electrophysiological analysis. Each band of the graph represents a recording session.

choice behavior on a T-maze. This procedure allowed a dissociation of the features associated with the current and the previous trials. We examined whether the firing of a single hippocampal cell can represent combinations of independent taskcontingent features. In addition, we tested if the task-contingent firing of place cells emerged via the activation of different place maps or through firing rate changes of spatially stable place cells. Finally, we investigated whether the coding mechanisms observed in this conditional discrimination task were also at play when the previous location of the animal guided the animal's behavior.

\section{Materials and Methods}

Subjects. Three male Long-Evans rats (weighting 285-310 g) were individually housed with ad libitum access to water. After a recovery period following tetrode implantation, but before behavioral training, the rats were put on a restricted feeding schedule to reduce their body weight to $\sim 85 \%$ of their free feeding weight.

Tetrode implantation. The rats were implanted with 16 tetrodes above the dorsal hippocampus. Each tetrode was made of four $12 \mu \mathrm{m}$ tungsten wires (H-Formvar insulation with Butyral bond coat, California Fine Wire Company), twisted together, and heated to bind them into a bundle. The tip of each wire was gold plated to reduce its impedance to $150-400 \mathrm{k} \Omega$. The tetrodes were loaded into a microdrive that allowed their independent movement. The rats were anesthetized with isoflurane (0.5-2\%) mixed with oxygen, injected intraperitoneally with buprenorphine $(0.1 \mathrm{mg} / \mathrm{kg})$, and positioned in a stereotaxic apparatus. The skull was exposed. Bregma and lambda were leveled and 9-10 anchor screws were affixed to the skull. A craniotomy was performed above the right dorsal hippocampus, $3.0-4.6 \mathrm{~mm}$ posterior and $2.2-3.8 \mathrm{~mm}$ lateral to bregma. The dura mater was removed and the tetrodes were lowered 1.5 $\mathrm{mm}$ into the cortex, after which both the tetrodes and the exposed cortex were sealed with paraffin wax. Two copper wires were attached to two of the anchor screws above the cerebellum and served as ground and reference electrodes. The microdrive was permanently attached to the skull and the anchor screws using dental acrylic. Following a $7 \mathrm{~d}$ recovery period, the tetrodes were lowered until they were $\sim 300$ $500 \mu \mathrm{m}$ above the CA1 pyramidal cell layer.

Apparatus. The modified T-maze was made of wood and painted gray (Fig. $1 A$ ). The maze, which was elevated $76 \mathrm{~cm}$ from the floor, consisted of a standard T-maze to which two additional return arms were attached between the goal arms and the start of the central arm. The central arm was $162 \mathrm{~cm}$ long and each goal arm was $50 \mathrm{~cm}$ long. The maze arms were $10 \mathrm{~cm}$ wide. A 1-cm-high rim secured the edge of each alley. Inverted guillotine doors that dropped down below the maze were located $2.5 \mathrm{~cm}$ away from beginning and end of the central arm on both sides. The doors could be operated from a distance by the experimenter who pulled or released strings connected to the doors. A food well was located on the central arm, $30 \mathrm{~cm}$ away from beginning of that arm. A food well was also located at the end of each of the goal arms. The room in which the T-maze was located was illuminated by 6 light bulbs attached to a circular ring above the maze. The testing room contained many extramaze visual cues that remained at the same location throughout testing, including two computer racks, a black curtain, a door, and shelves.

Behavioral procedure. Rats were familiarized to the maze individually by placing them on the maze for $10 \mathrm{~min}$ twice a day. On each session, sweet corn or chocolate was available in the food well of the central arm and in one of the two goal arm food wells. For example, on one familiarization session, an animal was familiarized with chocolate in the food well of the central arm and the left goal arm. On the next session, this animal was then familiarized with sweet corn in the food well of the central arm and of the right goal arm. This way, each goal arm food well was associated with a particular type of food. This contingency remained the same for a given animal throughout training but varied across animals. After the third day, the guillotine doors at the entrances of the goal arms and at the entrance of the base of the central arm (Fig. 1A) were opened and closed during the familiarization sessions.

Training on the continuous conditional discrimination task began after $4-5 \mathrm{~d}$ of familiarization. A session would start when the rat was placed on the base of the central arm. The rat was allowed to eat the food reward (chocolate or sweet corn) from the food well on the central arm. The rat was then required to run up the central arm and visit one of the two goal arms. The baited goal arm depended on the food type given to the animal at the base of the central arm. For example, if sweet corn was given to the animal on the central arm of the maze, more sweet corn was also available in the food well of the right goal arm. If chocolate was in the food well of the central arm, then more chocolate was available in the food well of the left goal arm. The other food well remained unbaited. Once the rat was in one of the goal arms, the guillotine door of this arm was closed to prevent the rat from visiting the other goal arm or retracing its steps to the central arm. The rat then had to run down the return arm to reach the base of the central arm and initiate the next trial. During the first 12 trials of a training session, the rat received two blocks of six consecutive trials with the same type of food to break the tendency of the rats to alternate between left and right turns. Subsequently, the rat was trained on 24 trials in which the identity of the food was established using a pseudorandom order in which the same food stuff was not repeated on $>3$ consecutive trials (with 12 chocolate trials and 12 sweet corn trials within each session). During this period, rats received, on average, two 
training sessions of 36 trials per day. As training progressed, the guillotine doors were used only when needed, usually $<5$ times per session. The recording sessions started after the rats had reached a criterion of at least $80 \%$ correct choices during one training session (i.e., 29 correct out of 36 trials). On recording sessions, the rats first received two blocks of six consecutive trials with the same food type, as during the training sessions. Thereafter, the order of the food types was determined pseudo-randomly (as above) and the rats were run until they were satiated.

Data acquisition and analysis. The signals from the tetrode wires were passed through two 32 channel unity-gain preamplifier panels (http:// www.braintelemeter.atw.hu) to reduce cable artifacts. The output signals were amplified $(0.1 \mathrm{~Hz}$ to $5 \mathrm{kHz}$, amplification: $\times 1000,64$ channel Sensorium Amplifier) and continuously digitized at $20 \mathrm{kHz}$ using AD converter computer cards (United Electronics Industries).

At the beginning of each recording day, the tetrodes were lowered into the pyramidal cell layer of the hippocampus and recording sessions began 3-4 h later. At the end of a recording day, the tetrodes were raised $\sim 500 \mu \mathrm{m}$.

Spikes were extracted offline from the digitally filtered $(0.8-5 \mathrm{kHz})$ signal. The power (root mean square) of the filtered signal was computed in a sliding time window $(0.2 \mathrm{~ms})$ and the SD was calculated to estimate the variance of the baseline noise. The time windows with a power of $>5$ times the SD from the baseline mean were extracted. The 32 samples (1.6 ms) containing each spike were reconstructed at $40 \mathrm{kHz}$ using the principles of the sampling theorem (Press et al., 2007). The peaks of the original and reconstructed waveforms were realigned. Sixteen data points were then extracted for each spike (assuming $10 \mathrm{kHz}$ ). Principal component analysis was used to extract the first three components of the spike waveforms for each wire of the tetrode. Normally, the first three principal components account for $>90 \%$ of the variance. For each spike, the three principal components for each wire were combined, giving a total of 12 features for each spike.

The spikes from putative individual neurons were segregated using automatic clustering software (http://klustakwik.sourceforge.net/) and the resulting spike clusters were manually refined with a graphical cluster cutting program. Only clusters with well-defined cluster boundaries and a clear refractory period in their spike-time autocorrelation were used for further analysis. Pyramidal cells and interneurons were discriminated by their spike-time autocorrelation, firing rate and waveform, as previously described (Csicsvari et al., 1999). Stability of the cells was verified by analyzing spike features over time. In addition, an isolation distance, based on Mahalanobis distance, was calculated to ensure that spike clusters did not overlap during the course of the recordings. Clusters with $<300$ spikes were not used in further analysis.

For each rat, we used one of the tetrodes in the CA1 pyramidal cell layer to detect theta oscillations. The raw signal was digitally bandpass filtered at delta $(2-4 \mathrm{~Hz})$ and theta $(6-10 \mathrm{~Hz})$ frequency and the power (root mean square) was calculated for every $500 \mathrm{~ms}$ time window. Theta epochs were defined as time windows with a theta/delta power ratio $>1.5$. The raw signal was then filtered at $5-14 \mathrm{~Hz}$ and the beginning and end of each cycle in the theta epochs were detected. Phase "zero" was defined as the positive-to-negative zero-crossing. The firing phase of a spike in degrees was the elapsed fraction of the current cycle at the time of the spike, scaled to a $0-360$ range.

Sharp wave/ripple epochs were detected on tetrodes located in the CA1 pyramidal cell layer after applying a bandpass filter $(125-250 \mathrm{~Hz})$ to the raw signal. A channel from a tetrode located outside the CA1 pyramidal cell layer served as a reference channel to eliminate contamination of the detection by muscle artifacts. The power (root mean square) was calculated in $20 \mathrm{~ms}$ time windows ( $10 \mathrm{~ms}$ between windows) and a window with a power larger than $5 \mathrm{SD}$ above the mean was considered as part of a sharp wave/ripple epoch. The epoch extended in both directions until a time window with a power below 2.5 SD above the mean was encountered.

The position of the animal was obtained by monitoring the position of three LEDs of different colors located on the animal's microdrive via a video camera (Digital 8, Handycam, Sony) mounted in the ceiling above the maze. The position of the animal was sampled every $40 \mathrm{~ms}$ and the resolution of the tracking was 3.3 pixels $/ \mathrm{cm}$.

Place rate maps were calculated by dividing the number of spikes emitted by the time spent in $2 \times 2 \mathrm{~cm}$ bins. Only the data from periods in which the velocity of the rat was higher than $5 \mathrm{~cm}$ per second were included in the calculation. Both the occupancy (time spent in each bin) and firing rate maps were smoothed with a Gaussian kernel function $(\mathrm{SD}=3 \mathrm{~cm})$.

For some analyses, the two-dimensional position data were transformed into one dimension. This was done by finding the seven linear vectors that best represented the path of the animal on each of the seven segments of the maze (i.e., the two oblique portions of the return arms, the two horizontal portions of the return arms, the central arm and the two goal arms). For each position sample, the point ( $p$ ) on these vectors that was the closest to the position of the animal was found. The distance from the end of the oblique return arm close to the food well and $\mathrm{p}$ represents the linear position of the animal. The length of the linear path for a typical trial was $\sim 400 \mathrm{~cm}$. A linear place field was defined as a minimum of five adjacent $2 \mathrm{~cm}$ bins containing a bin with a firing rate above $5 \mathrm{~Hz}$, and extending both sides until the firing rate fell below $10 \%$ of the highest firing rate in the field.

Influence of trial type and previous location on the firing of hippocampal pyramidal cells. Each correct trial was classified according to two factors: the conditional cue given to the animal (sweet corn or chocolate) and the previous location of the animal before entering the central arm of the maze (right or left return arm). The firing rate of every pyramidal cell on each trial was calculated in two zones of the central arm of the maze. The conditional cue zone $(\mathrm{CCZ})$ was a $25 \times 25 \mathrm{~cm}$ area centered on the food well of the central arm (Fig. 1A). The middle central arm zone (MCAZ) was a rectangular $25 \times 90 \mathrm{~cm}$ area covering the central arm, starting 20 $\mathrm{cm}$ from the food well of the central arm (Fig. $1 \mathrm{~A}$ ).

For the CCZ analysis, the time spent in the CCZ on each trial was divided into four time windows of equal duration. The average speed and the $X$ and $Y$ positions of the animal were calculated for each time window, together with the firing rate of every cell that emitted $>300$ spikes in the CCZ. An analysis of covariance (ANCOVA) on the firing rate with the factors Trial-Type (correct sweet corn trials and correct chocolate trials), Previous-Location (left or right return arm), Time-Window (4 equal time windows), and the continuous variables X-Pos, Y-Pos, Speed, and Time-In-Zone was performed. This aimed to establish whether the factor Trial-Type, Previous-Location, and Time-Window had an effect on the firing rate of a cell once the effects of X-Pos, Y-Pos, Speed, and Time-InZone had been removed. A shuffling procedure was used to minimize the risk that a deviation from a Gaussian distribution in the firing rate of the cells affected the conclusions of the statistical procedure. The ANCOVA was repeated 500 times for each cell after shuffling the identity of the Trial-Type and Previous-Location. The significance level for the different effects for a given cell was obtained by comparing the real $F$ value with the distribution of $F$ values obtained during the shuffling procedure on the data of that same cell. If the real $F$ value was larger than $95 \%$ of the $F$ values obtained during the shuffling procedure, the effect was considered statistically significant. At the cell population level, we calculated the mean $F$ value for the factors Trial-Type and Previous-Location from the real data set. Five-hundred samples of size $n$, where $n$ is the number of neurons analyzed, were taken from the shuffled distribution of $F$ values (containing the shuffled data from all analyzed cells), and the mean $F$ value of each sample was calculated. If the mean $F$ value from the real data set was larger than $99 \%$ of the mean $F$ values observed during this shuffling procedure, we concluded that the factor was significant at the population level. We also calculated $95 \%$ intervals for a shuffled cumulative distribution of $F$ values. This was done by calculating the cumulative distribution of each of the 500 samples of size $n$, and keeping the 25th largest probability for each value of $F$.

A differentiation index was calculated for each cell in the CCZ zone. This score was defined as the absolute value of the difference between the mean firing rates on the two types of trials (food types), divided by the sum of mean firing rates on the two types of trials. We also calculated a differentiation index for the same cells using the firing at the food wells in the right and left goal arm. 
To test whether the factors Trial-Type and Previous-Location had an effect on the linear place rate maps in the MCAZ, a linear place rate map of the MCAZ was calculated on each trial for every cell. Each bin represented $8.2 \mathrm{~cm}$ of the MCAZ. The mean speed and lateral position (position along the shortest axis of the MCAZ) of the animal was also estimated in each individual bin of every trial. An ANCOVA was performed for each cell to determine whether the factors Trial-Type, Previous-Location, and Position had an effect on the firing rate of the cell, while controlling for the potential effects of speed and the lateral position of the animal. We repeated this calculation 500 times after shuffling the identity of the correct trials. The distribution of $F$ values for the factors Trial-Type and Previous-Location from the real data was compared with that of the shuffled data set.

Place field similarity in different trial conditions. To examine whether the location and shape of the place fields changed depending on the trial type and/or the previous location of the animal, two-dimensional place rate maps within the MCAZ were made from the trials with chocolate or sweet corn, or from the trials on which the animal was coming from the right or left return arm. For each selected cell, a Pearson correlation coefficient was calculated between the firing rates of two place rate maps across two different conditions. The distribution of correlation coefficients was compared with a similar distribution obtained after shuffling the trial identity 500 times.

Theta oscillations and phase precession analysis. For place cells showing changes in firing associated with the trial type or the previous position of the rat, the theta-phase precession profile was quantified in the two conditions showing a firing rate difference. Place-phase maps were calculated in a similar manner to those of the linear place rate maps, but the maps included only the region covered by the place field of the cell plus a $10 \mathrm{~cm}$ margin area. The spike-count maps (as a function of the rat's location on the linear track and theta phase) and their associated occupancy maps were calculated (equal occupancy probability being assumed for different theta phases). These maps were calculated separately for the types of trials in which there was a change of firing rate. As different cells had a higher firing rate in different conditions, the group data are reported for the trial conditions associated with low and high firing rate. Theta phase precession was quantified by calculating the $r$ value of the circular-linear correlation between the theta phase and linear position of the spikes. The mean phases of the spikes for six equal portions of the cell's place field were also calculated.

Position reconstruction from ensemble of pyramidal cells. The rat's position was reconstructed using a cross-validation method to test whether the different trial types induced spatial remapping in the hippocampus. The method was adapted from previous work in which the activity of pyramidal cells was used to predict the location of the rat on a linear track or a figure-8 maze (Jensen and Lisman, 2000; Zhang et al., 1998). The MCAZ was divided in $4 \times 4 \mathrm{~cm}$ bins and the Bayes's theorem was used to calculate the probability that the rat was at position $x$, given the number of spikes $(n)$ observed in a $200 \mathrm{~ms}$ time window, as follows:

$$
P(x \mid n)=\frac{P(n \mid x) P(x)}{P(n)} .
$$

$P(x)$ is the probability that the rat was at position $x . P(n \mid x)$ was derived using the firing rate maps of the pyramidal cells in the MCAZ, as follows:

$$
P(n \mid x)=\prod_{i=1}^{N} P\left(n_{i} \mid x\right)=\prod_{i=1}^{N} \frac{\left(\tau f_{i}(x)\right)^{n_{i}}}{n_{i} !} \exp \left(-\tau f_{i}(x)\right)
$$

where $f_{i}(x)$ is the mean firing rate of cell $i$ at position $x$ and $\tau$ is the length of the time window in seconds. The probability $P(n)$ was determined by normalizing the conditional probability $P(x \mid n)$ so that the $\operatorname{sum}$ of $P(x \mid n)$ over $x$ was equal to 1 . Hence, $P(n)$ was calculated by summing $P(n$ $x) P(x)$ for all possible $x$ values. The model used to predict the position of the rat on a given trial was based on the place rate maps of the cells during the remaining trials of the same type or all but one randomly selected trial of the different type. For each time window, the position $x$ associated with the largest probability was the reconstructed position. If in a time window the sum of $P(x \mid n)$ over $x$ was equal to 0 , the predicted position of the rat was not updated. Theta phase information was added to the model by dividing each theta cycle into five theta bands (Jensen and Lisman, 2000). This was done by calculating the firing rate maps separately for the spikes falling in the different theta bands (smoothing kernel $\mathrm{SD}=8 \mathrm{~cm}$ ). The reconstruction error for each $200 \mathrm{~ms}$ time window was defined as the distance in the longitudinal axis of the central arm between the recorded and predicted positions. To estimate the effect that global remapping would have on the reconstruction error, we reconstructed the position of the rat after shifting the position of the firing fields in the model. The fields were shifted by moving the firing rate map along the longitudinal axis of the MCAZ by a random amount, imposing circular boundary conditions.

Prediction of trial-related information based on ensemble firing rates. The firing rate of hippocampal cells in the CCZ and the MCAZ was also used to predict the trial type or the previous location of the rat. In the CCZ, the firing rate of each cell with a significant main effect of TrialType or Previous-Location was included as a predictor. For cells involving a significant interaction with Time-Window, the firing rate in the time windows in which there was a significant simple main effect of Trial-Type or Previous-Location served as a predictor. In the MCAZ, the firing rates in the linear place rate maps were used. For cells with a significant main effect of Trial-Type or Previous-Location, but no significant interaction with the factor Position, the average firing rate in the place rate maps served as predictors. For the cells with a significant interaction involving the factor Position, simple main effects were calculated for each bin of the linear place maps. The firing rates in the bins with a significant simple main effect of Trial-Type or Previous Location were used as predictors.

To infer the trial type and the previous location for a given trial, we fit the model on all but one trials in the same recording session (training set) and used the model to predict the trial type and the previous location of the remaining trial (Pastalkova et al., 2008). For each trial of the training set, a binary population vector $u=\left(u_{1}, u_{2}, u_{3} \ldots u_{k}\right)$, where $k$ is the number of predictors, was constructed. Each predictor could be in one of two states: $u_{k}=$ high if $n_{k}<N_{k}$ and $u_{k}=$ low if $n_{k}<N_{k}$, where $n$ is the firing rate of the predictor on the trial and $N$ its mean firing rate across all trials of the training set. The mean firing rate of the cell was used as the discriminator because the predictors were identified from the results of the ANCOVA, which tested whether the mean firing rate of the predictor varied between different conditions. Four population vectors were used to store the probability for each predictor being in the high or low states, on each of the four possible combinations of trial types and previous locations:

$P_{q}^{k}(u)=\operatorname{Prob}(k$-th predictor is in the state $u$ given trial type and previous location $q$ ).

The high or low state of each predictor was then established for the predicted trial and the probability of obtaining this vector was computed using the following formula:

$$
P_{q}(u)=\prod_{k=1}^{K} P_{q}^{k}\left(u_{k}\right) .
$$

The condition with the largest probability was defined as the predicted condition.

Rewarded alternation without delays. An additional four, experimentally naive adult rats were implanted with 16 tetrodes in the hippocampus and trained on the continuous version of the rewarded alternation task without delays (Wood et al., 2000; Lee et al., 2006). The same apparatus was used as for the conditional discrimination task. Two food wells were used in this experiment. They were located at the distal ends of each goal arm. Two pellet dispensers (Med Associates) located in the ceiling above the maze were used to deliver food pellets $(45 \mathrm{mg}$, Formula A/I, P. J. Noyes) to each food well via a plastic tube.

The rats were first trained to run unidirectional laps on one side of the maze (either the left or the right side). The access to the opposite side of the maze was blocked by two black barriers $(40 \mathrm{~cm}$ wide and $50 \mathrm{~cm}$ high). During the first two days, pellets were scattered on the maze and the rat was allowed to explore half of the maze for $10 \mathrm{~min}$. On the following days, 
each rat was given two 10 min sessions on the maze and pellets were only available at the food well. A food reward was delivered each time the rat completed a lap on the maze and visited the food well in the goal arm. The rat then had to return to the start of the central arm via the return arm. The maze doors were used to prevent the animal from retracing its steps. Once the rat performed unidirectional laps without much hesitation, the recording cable was attached to the rat before it was placed on the maze. After 3-4 d of unidirectional lap training on the same side of the maze, the rats were trained for $1 \mathrm{~d}$ to perform unilateral laps on both the familiar and novel sides of the maze. Unilateral training sessions of 20 min were performed in this order: familiar-novel-familiar-novel. The access to the other arm was blocked with the barriers. Before each training session, the rat was placed for $20 \mathrm{~min}$ in a gray box $(30 \times 26 \mathrm{~cm}, 53 \mathrm{~cm}$ high). The rewarded alternation training without delays began the following day.

During rewarded alternation training, the barriers were removed from the maze and the rat was free to alternate between the left and right goal arms of the maze. A food reward was available at the food well whenever the rat visited the goal arm opposite to the one it had visited on the previous trial. The doors of the maze were used only to prevent the rat from retracing its route or entering a return arm via the base of the central arm.

The data from the left-to-right, right-to-left, and error trials were separated using the position data. Place field analysis was performed as for the conditional discrimination task. The analysis of the effect of trial type (left-to-right and right-to-left trials) on the firing of the cells was performed on the central arm of the maze. The zone of analysis began $30 \mathrm{~cm}$ away from the return arm junction with the central arm and extended $115 \mathrm{~cm}$ along the central arm. The position of the rat in that zone was very similar across the two trial types.

Histology. After completion of the experiment, rats were deeply anesthetized (sodium pentobarbital, $200 \mathrm{mg} / \mathrm{kg}$, i.p.) and perfused transcardially with $0.9 \%$ buffered saline, followed by $4 \%$ paraformaldehyde $\left(4^{\circ} \mathrm{C}\right)$. Brains were excised and stored in a solution of $4 \%$ paraformaldehyde. They were then placed in a sucrose-formalin solution for $24 \mathrm{~h}$, frozen, and sectioned $(50 \mu \mathrm{m})$ coronally. Sections containing the hippocampal region were mounted on a gelatin-coated slide, dried at $37^{\circ} \mathrm{C}$, and stained with cresyl violet. The sections were examined to identify the location of the tetrodes.

\section{Results}

Multichannel extracellular recordings were performed in the dorsal hippocampus during the conditional discrimination task. The majority of the tetrodes $(>80 \%)$ were located in the CA1 region, with the remaining tetrodes located near the CA3 and CA2 border. We identified 532 pyramidal cells and 67 interneurons. Before recordings, rats were trained to perform the conditional discrimination task in which the animal had to turn left or right on the T-maze depending on the type of food reward it received in the central arm. After $\sim 500$ trials, the rats were performing consistently above $75 \%$ correct choices (Fig. $1 B$ ). The mean performance during subsequent recording sessions was $84.53 \%$ correct choices, with only one session below $80 \%$ correct choices (Fig. 1C). The performance on trials with chocolate and sweet corn was not significantly different, $F_{(1,12)}=1.36 ; p=0.73$ (Fig. 1C).

Most pyramidal cells had one or few defined firing fields on the maze (Fig. $2 \mathrm{~A}$ ). The mean linear place field size for pyramidal cells was $31.79 \pm 0.68 \mathrm{~cm}$. Place fields appeared stable during recording sessions and we did not find evidence for a gradual shift forward of the place fields within recording sessions, either on the central arm of the maze (Fig. $2 B$, effect of block: $F=0.9951, p=$ 0.45 ) or on the return arms (Fig. $2 C$, effect of block: $F=0.39, p=$ $0.85)$. The firing of pyramidal cells was examined in two locations on the central arm of the maze: the CCZ and the MCAZ, see Figure $1 A$.
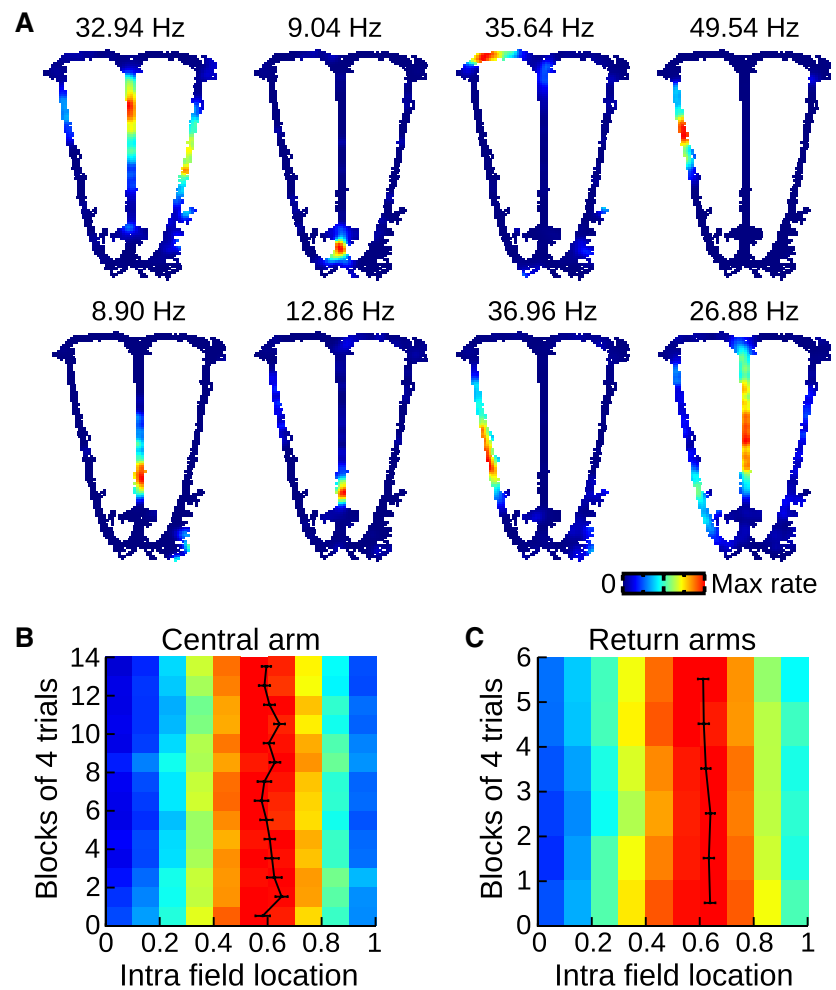

Figure 2. Place rate maps of pyramidal cells during a conditional discrimination task. $\boldsymbol{A}$, Examples of place rate maps from eight pyramidal cells recorded simultaneously on the modified T-maze. The number above each map is the peak firing rate of the cell. $\boldsymbol{B}$, Stable firing fields during the conditional discrimination task. The density plot shows the mean within-field firing rate (normalized) for blocks of four trials. Only fields located on the central arm were included. Block 0 represents the first four trials of the recording sessions. The black line indicates the mean location of the peak firing rates for each block of trials ( \pm SEM). $\boldsymbol{C}$, Same as $\boldsymbol{B}$ but for firing fields located on the return arms of the maze.

\section{Presentation of the conditional cues leads to changes in firing rate}

A total of 199 pyramidal cells fired at least 300 spikes each while the rats were in the CCZ (Fig. 1A). During that period, the rat arrived at the food well and was presented with one of the two food types, which determined whether the rat should choose the left or the right goal arm to obtain a further reward. For each cell, we performed an ANCOVA with Trial-Type (sweet corn or chocolate), Previous-Location (right or left goal arm), and TimeWindow (time in the CCZ was divided into 4 equal time windows) as main factors and X-Pos, Y-Pos, Speed, and TimeIn-Zone as covariates. The significance associated with each effect was obtained from a distribution of $F$ values generated during a shuffling procedure. This procedure was performed because the distribution of firing rates for most cells deviated significantly from the Gaussian distribution even when the three main factors were taken into account (Shapiro-Wilk test, 196 out of 199 cells).

Forty-six neurons $(23.12 \%)$ showed either a significant effect of Trial-Type or a significant Trial-Type versus Time-Window interaction, but no significant effect involving Previous-Location (Fig. $3 A, B$ ). Therefore, these cells changed their firing rate to reflect the trial type on any given trial. These types of cells were recorded in all recording sessions, ranging from 5.56 to $44.44 \%$ of the total number of recorded cells. The mean $F$ value for the Trial-Type effect in the real data set was larger than that obtained after shuffling the identity of the trials. (Fig. 3C, mean real $F$ value: 3.94 , smallest mean $F$ value in 


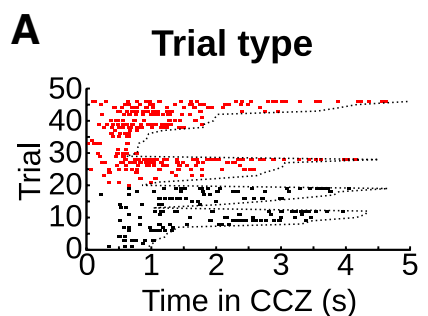

- Chocolate - Sweet corn

C

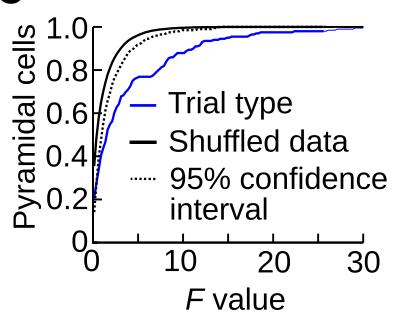

D Previous location

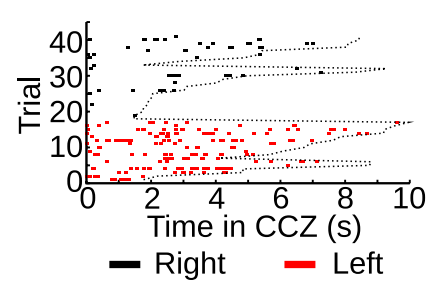

$\mathbf{F}$

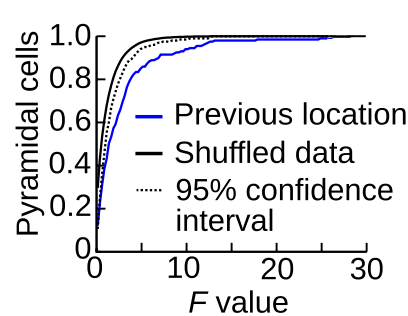

G

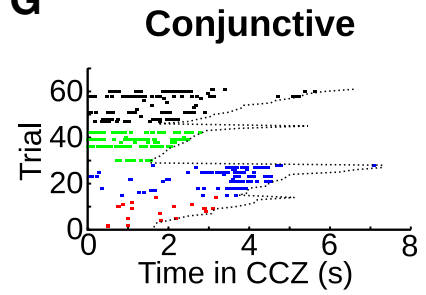

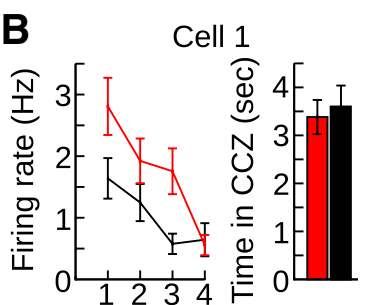

Time window

Cell 3

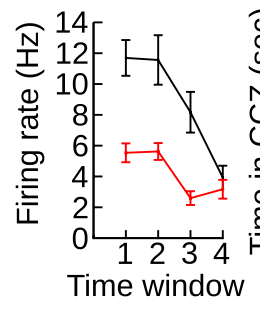

E

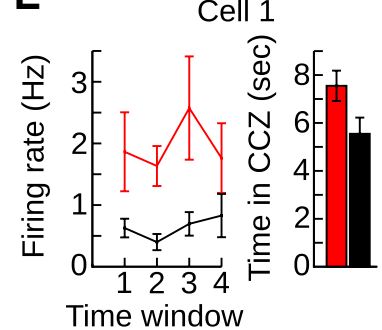

Cell 3

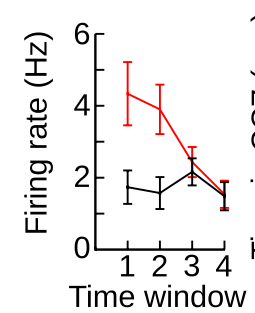

H Cell 1

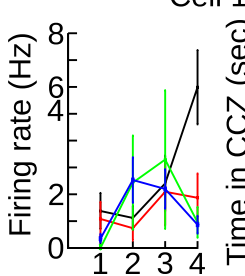

- Choco-right - Sweet corn-right Time window

- Choco-left - Sweet corn-left

I

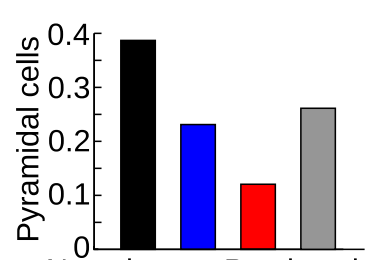

— Non sign. - Previous location

- Trial type - Conjunctive
Time window

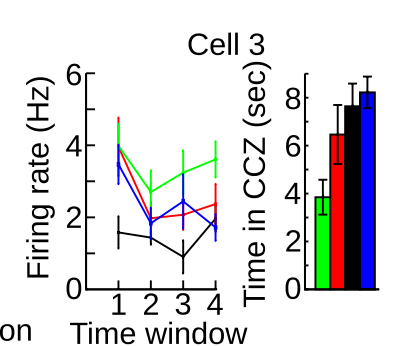

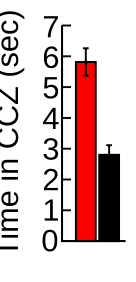
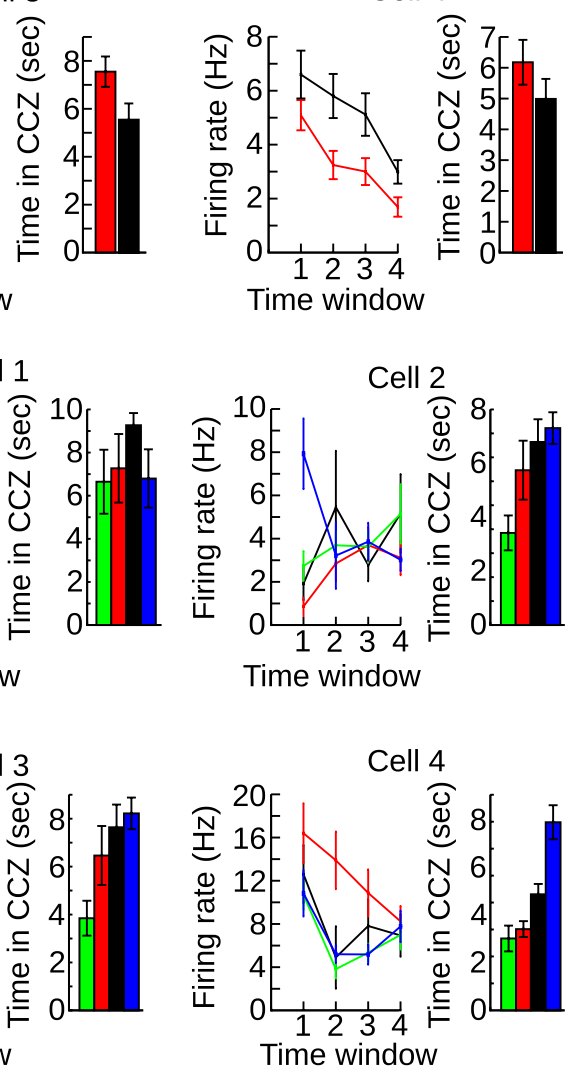

Figure 3. Effects of trial type and previous location in the CCZ. A, Raster plot of a pyramidal cells showing the spikes emitted while the rat was in the CCZ. The trials were (Figure legend continues.) 
shuffled samples with a probability $<0.01: 1.89$ ). This indicates that the effect of Trial-Type on the firing rate of the cells was not due to random fluctuations.

In separate ANOVAs, the effect of Time-Window on the firing rate in the CCZ was tested. The firing of 138 out of 199 cells $(69.35 \%)$ was significantly affected by the factor Time-Window. The variables X-Pos and Y-Pos affected the firing rate of a similar number of cells (136 out of 199 cells, 68.34\%).

The effect of Trial-Type on the firing rate of the cells was confirmed by a second analysis in which we calculated the distribution of differentiation indices for the 199 cells. Differentiation index was calculated as the absolute value of the difference between the mean firing rates on the two types of trials, divided by the sum of mean firing rates on the two types of trials. This calculation was repeated after shuffling the trial identity. The mean differentiation index in the real data set was larger than that obtained after shuffling the identity of the trials (mean differentiation index: 0.15 , smallest mean differentiation index from shuffled samples with a probability <0.01: 0.11$)$. Thus, larger differentiation indices were more frequent in the recorded data than in the shuffled data.

Cells with a significant effect of Trial-Type could be place cells for which the firing rate is modulated by the identity of the conditional cue, or they could be cells responding to a particular non-spatial cue (Wood et al., 1999). If the cells showing different firing rate at the central arm food well encoded the identity of the food independently of the position of the animal, then these cells should also exhibit similar food type-related firing at the food wells in the goal arm. To test this possibility, we calculated a second differentiation index between the firing rates of the cells at the goal arm food wells and compared it to that for the CCZ. There was no relationship between the differentiation indices in the CCZ and those in the goal arm reward sites $(r=0.09$; $p>$ $0.5)$. Thus, the fact that cells changed their firing rate depending on the conditional cue on the central arm of the maze did not simply reflect the encoding of information relative to the type of food independent of spatial location.

\section{The previous location of the rat influences firing rate in the CCZ}

Depending on the response of the rat on the previous trial, the rat could enter the CCZ after having run down either the left or right

\footnotetext{
$\leftarrow$

(Figure legend continued.) sorted according to the two conditions (trial type and previous location). Within these conditions, the trials were sorted according to the time the rat spent in the CCZ. The bars representing the spikes are color-coded according to the trial type of each trial. The dotted line indicates the time at which the animal left the CCZ on each trial. $\boldsymbol{B}$, The firing rate offour pyramidal cells that fired differently depending on the two types of food (trial type) in the $\mathrm{CCZ}$. The time in the $\mathrm{C} Z \mathrm{Z}$ on each trial was divided in four equal time windows. For each cell, the mean time in the CCZ for trials of the two conditions is shown. C, Cumulative distributions of the $F$ values obtained for the effect of trial type during the recording sessions (Trial type) and those obtained after shuffling the identity of the trials (Shuffled data). The dotted line indicates the $95 \%$ confidence intervals, which were calculated during the shuffling procedure (see Materials and Methods). Larger Fvalues were seen in the recording sessions as compared with what would be obtained by chance. $\boldsymbol{D}$, Previous location modulates firing rate. Raster plot of the spikes from a pyramidal cell in the CCZ. The bars representing the spikes are color-coded according to the previous location of the animal on each trial. $\boldsymbol{E}$, Four cells showing a significant effect of the previous location of the animal on the firing rate when the rat was in the $C C Z$. For each cell, the mean time in the CCZ for trials of the two conditions is shown. $F$, Cumulative distributions of $F$ values as shown in C but now for the effect of the previous location. $G$, Conjunctive effects of trial type and previous location. Raster plot showing the spikes of a cell on trials sorted according to the trial type and the previous location. $\boldsymbol{H}$, Four cells showing significant changes in firing rate related to both the trial type and the previous location of the animal. For each cell, the mean time in the CCZ for trials of the four conditions is shown. $I$, Proportion of the pyramidal cells with a significant effect of trial type, previous location, or both (conjunctive), on their firing rate in the $\mathrm{CCZ}$.
}

return arm. The previous location of the animal could affect the firing rate of pyramidal cells on the central arm, as previously shown during other memory tasks (Ainge et al., 2007b; Ferbinteanu and Shapiro, 2003; Pastalkova et al., 2008; Wood et al., 2000). The ANCOVA indicated that the firing of 24 neurons out of 199 (12.06\%) showed either a significant effect of Previous-Location or a Previous-Location versus Time-Window interaction, but no significant effect of Trial-Type or other interactions (Fig. 3D,E). The distribution of $F$ values for the effect of Previous-Location in the real data was compared with that obtained after shuffling the identity of the trials (Fig. 3F). The mean $F$ value for the Previous-Location effect in the real data was larger than what could be obtained by chance (mean real $F$ value: 2.92 , smallest mean $F$ value in shuffled samples with a probability $<0.01: 1.79)$. Therefore, a significant proportion of cells changed their firing rate in the CCZ depending on the previous location of the animal. It should be noted that the previous location of the rat is not only an indication of the journey of the rat before entering the CCZ, but is also related to the conditional cue presented on the previous trial.

A discrimination index for the previous position was also calculated. The mean differentiation index in the real data set was larger than that obtained after shuffling the identity of the trials (mean differentiation index: 0.14, smallest mean differentiation index from shuffled samples with a probability $<0.01$ : 0.11).

\section{Cells influenced by both the trial type and the previous location of the animal}

ANCOVAs performed on pyramidal cells firing in the CCZ showed that 52 cells $(26.13 \%)$ were influenced by both the TrialType and Previous-Location factors, or by an interaction involving these two factors, with or without the factor Time-Window (Fig. 3G,H). This shows that a significant proportion of hippocampal cells showed changes in firing rate associated with both the trial type and the previous location of the rat. For example, the raster plot of one cell in Figure $3 G$ showed a different firing pattern during trials with sweet corn, depending on whether the rat was coming from the left or right goal arm. The mean $F$ value for the Trial-Type $\times$ Previous-Location interaction in the real data was larger than what could be obtained by chance (mean real $F$ value: 1.99 , smallest mean $F$ value in shuffled samples with a probability $<0.01: 1.63)$. This confirmed that the firing of a significant proportion of cells was affected by both the trial type and the previous location of the animal. Figure $3 I$ shows the proportion of the recorded cells that were affected by the trial type, the previous location, or by a combination of these two factors (conjunctive).

We then assessed how conditional firing associated with the features of the task evolved when the rat was in the CCZ. An ANCOVA with Trial-Type and Previous-Location as main factors and X-Pos, Y-Pos, Speed, and Time-In-Zone as covariates was performed separately for the first and fourth time windows in the CCZ. The $F$ values obtained in the first time window were significantly larger than that in the fourth time window for both factors (Trial-Type, first time window: $2.69 \pm 0.30$, last time window: $1.10 \pm 0.10, p<10^{-05}$; Previous-Location, first time window: $1.76 \pm 0.17$, last time window: $1.21 \pm 0.13, p=0.007$ ). This result suggests that conditional firing in the CCZ was stronger when the rat had just entered the zone than when the rat was about to leave it. 
A

\section{Trial type}
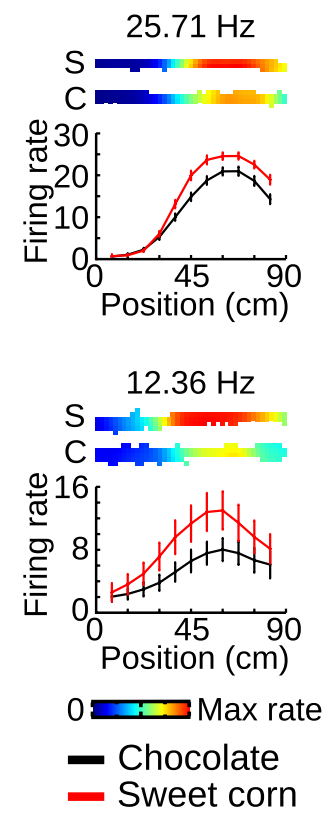

B

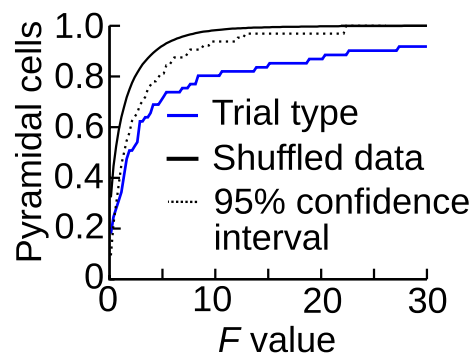

\section{Crevious location}
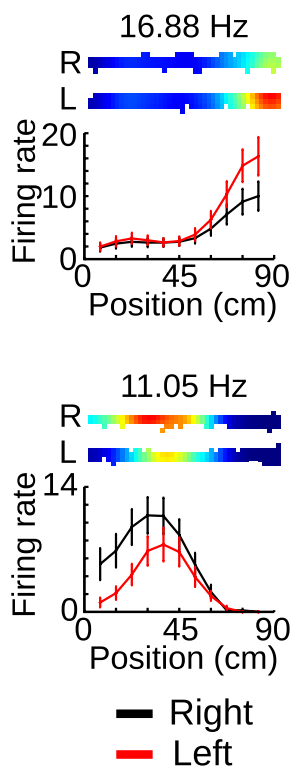

E
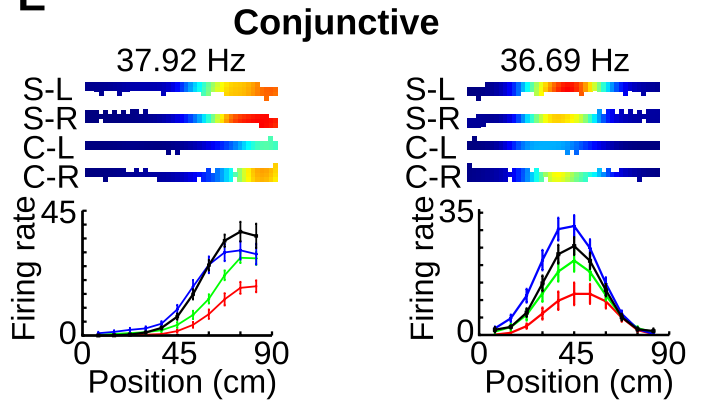

$13.95 \mathrm{~Hz}$

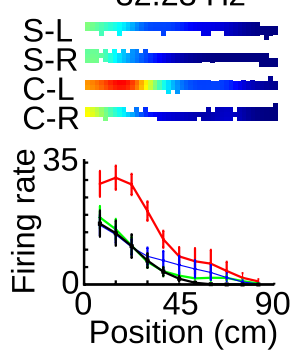

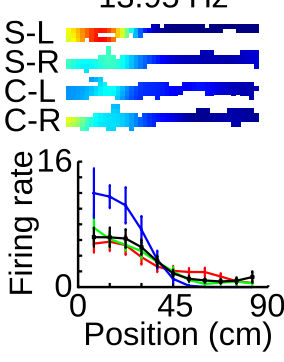

- Choco-right - Sweet corn-right - Choco-left - Sweet corn-left

D

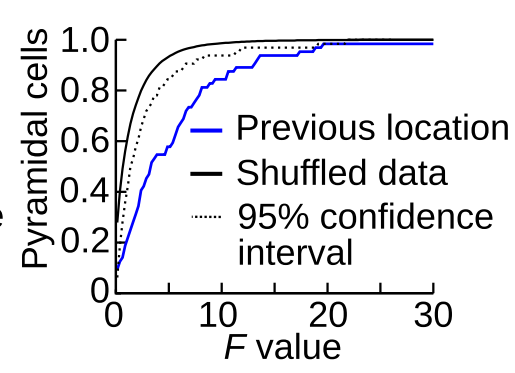

$\mathbf{F}$

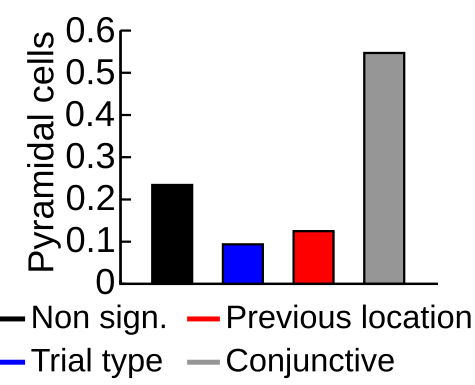

Figure 4. Effect of the trial type and previous location when the rat is in the MCAZ.A, Trial type modulation of firing rate. Examples of two pyramidal cells showing significant changes in the firing rate depending on the trial type. The density plots show place rate maps of the cells in the MCAZ for the two trial types (S, sweet corn; $C$, chocolate). The number above each density plot is the peak firing rate of the cell in the MCAZ. The graphs show the mean firing rate ( \pm SEM) of the same cells in a linear representation of the MCAZ. B, Cumulative distributions of the $F$ values obtained for the effect of trial type during recording sessions and those obtained after shuffling the identity of the trials (Shuffled data). $C$, The previous location of the animal affects firing rate in the MCAZ. The place rate maps of two pyramidal cells affected by the previous location of the animal ( $L$, left; $R$, right) are shown. $D$, Cumulative distributions of the $F$ values obtained for the effect of previous location during recording sessions and those obtained after shuffling the identity of the trials. $E$, Four cells affected by both the trial type and the previous location of the animal. The firing rate of the cells is shown for the four possible combinations of trials (C-L: chocolate, right; C-R: chocolate, right; S-L: sweet corn, left; S-R: sweet corn, right).F, Proportion of the pyramidal cells with a significant effect of trial type, previous location, or both (conjunctive), on the firing rate of the cells in the MCAZ.

Trial-related modulation of firing rate while the rat is running toward the choice point

After consuming the food reward in the CCZ, the rat ran toward the choice point of the maze. We used this part of the trial to assess whether the firing rate of pyramidal cells was also modulated by the trial type and/or the previous location of the animal while the animal was running. The analysis was limited to the MCAZ (Fig. 1A), where the behavior of the rat was very stereotyped and did not vary substantially depending on the trial type or the previous location of the animal. A total of 64 pyramidal cells fired at least 300 spikes in this section of the maze. These cells had a clear region of high firing on a limited section of the central arm of the maze (Fig. 4). An ANCOVA was performed for each cell to determine whether the factors Trial-Type, PreviousLocation, and longitudinal Y-Position had an effect on the firing rate of the cell, while controlling for the potential effects of speed and the lateral position of the animal. Six cells $(9.38 \%)$ showed either a significant effect of Trial-Type or Trial-Type versus
Y-Position interaction, but no significant Previous-Location effects or interactions involving this factor (Fig. 4A). The mean $F$ value for the effect of Trial-Type in the real data was larger than what could be expected by chance (Fig. $4 B$, mean $F$ value: 10.02 , smallest mean $F$ value in shuffled samples with a probability $<0.01: 3.27)$. The factor Previous-Location also had a significant effect on an additional eight cells (Fig. 4C). These cells showed a significant effect of Previous-Location or Previous-Location versus Y-Position interaction, but no significant Trial-Type effects or interactions involving this factor. The mean $F$ value for the Previous-Location factor was significantly different from chance levels (Fig. $4 D$, mean $F$ value: 5.44 , smallest mean $F$ value in shuffled samples with a probability $<0.01: 3.03)$. Nevertheless, most cells showing changes of firing rate related to the task were influenced by both the trial type and the previous location of the animal (Fig. $4 E$ ). Thirty-five cells (54.69\%) showed either significant effects of Trial-Type and Previous-Location or at least one significant interaction involving both these two factors. As can be 
seen on Figure $4 E$, most of these cells had stable place fields across the four conditions, but the precise firing rate of the cells within their fields varied depending on trial type and previous location. For example, one cell presented on Figure $4 E$ shows an elevated firing rate specifically for the trials in which the rat had been presented with sweet corn and was coming from the left. Again we tested whether these results were significantly different from what could be obtained by chance by comparing the $F$ values for the interaction Trial-Type versus Previous-Position with that of the same interaction after shuffling the trials. The mean $F$ value in the data set was significantly larger than that obtained after shuffling the data (mean $F$ value: 6.01, smallest mean $F$ value in shuffled samples with a probability $<0.01: 1.75)$. Figure $4 F$ summarized the proportion of the cells showing different effects in the MCAZ.

\section{Trial-related coding is present during periods of reduced theta activity}

Retrospective and prospective coding in the hippocampus has been observed mainly during running episodes (Wood et al., 2000; Ferbinteanu and Shapiro, 2003; Pastalkova et al., 2008). These periods are characterized by the strong modulation of the firing of pyramidal cells and interneurons at theta frequency. In the current experiment, trial-related firing was also observed as the animal paused to obtain a food reward and as it was running toward the choice point. We therefore investigated whether theta activity modulated the firing of pyramidal cells and interneurons in a similar fashion in the two phases of the trials. The detection of theta cycles was limited to epochs where a clear increase of power at theta frequency was observed (theta/delta ratio $>1.5$, Fig. $5 A$ ). The theta/delta ratio was above this threshold during $\sim 60 \%$ of the recording sessions (Fig. 5B). A power spectrum was first calculated to determine if theta oscillations were present in the hippocampus while the rat was in the CCZ or in the MCAZ (Fig. $5 C$ ). Figure $5, D$ and $E$, shows that there was a significant reduction in the power at theta frequency when the animal was in the CCZ (paired $t$-tests; $p<0.01$ from 7.6 to $8.2 \mathrm{~Hz}$ ).

To quantify the theta modulation of the firing rate of pyramidal cells and interneurons in the two zones, we calculated the mean theta vector length for pyramidal cells and interneurons (Fig. $5 F$ ). The mean theta vector length for pyramidal cells was reduced in the CCZ compared with the MCAZ $\left(p<10^{-10}\right)$. In contrast, the mean theta vector length for interneurons was not significantly different across the two zones $(p>0.10)$. The theta phase preference of cells in the $\mathrm{CCZ}$ and the MCAZ was also investigated. When the animal slowed down to receive a food reward in the CCZ, pyramidal cells showed no clear preference for a particular theta phase (Fig. $5 G$ ). In contrast, as the animal ran in the MCAZ pyramidal cells tend to fire at the trough of the theta oscillations. The pattern of results was different in the interneuron population (Fig. 5G). They tended to fire at the descending phase of the theta oscillations in both the CCZ and the MCAZ. The mean spike-time autocorrelation of pyramidal cells also showed a clear reduction in the tendency to fire periodic spikes at theta frequency in the CCZ (Fig. $5 \mathrm{H}$ ), indicating that these cells do not periodically fire in subsequent theta cycles. Thus, they cannot participate accurately in theta phase precession in this condition. In contrast, the firing patterns of interneurons appeared more similar across the CCZ and the MCAZ. These results show that the firing patterns of pyramidal cells changed during different phases of the trials. Pyramidal cells, however, encoded information relative to the trial type and the previous location in both states.
It is possible that the presence of sharp wave/ripples during periods of relative immobility in the CCZ could have affected the detection of theta oscillations. However, we found that sharp wave/ripple epochs accounted for $0.24 \%$ of the time spent in the CCZ and only $0.58 \%$ of the theta epochs. Removing sharp wave/ ripple epochs from the analysis presented in Figure 5 had no visible effect on the results.

\section{Spatial coding is not affected by task-related modulations of firing rate}

Although the firing rate of place cells changed depending on the trial type and the previous location of the animal, the shape and location of their place fields were not affected. For the cells firing 300 spikes in the MCAZ, two-dimensional place rate maps within the MCAZ were constructed for both trial types and both previous locations. The correlation coefficients between the firing rates in these two maps were high (mean $r$ value $=0.81 \pm 0.02$ ). The distribution of the correlation coefficients was not significantly different from that obtained after shuffling the identity of the trials (Fig. $6 A$, all $p$ values $>0.05$ ). Similar results were obtained when smoothing kernels of different width were used to calculate the place rate maps (kernels with SD ranging from 0 to $4 \mathrm{~cm}$ ). This suggests that the location of the place fields did not change significantly depending on the trial types.

Hippocampal place cells also encode spatial information via changes in the theta phase of individual action potentials. We therefore assessed the profile of theta phase precession for cells showing firing rate changes associated with the current trial types or the previous location on the maze. For each cell showing an effect of Trial-Type, Previous-Location, or an interaction between these two factors in the MCAZ, the place-phase profile of its action potentials was constructed. Either the two placephase profiles were compared, or, if significant interactions were detected, conditions associated with the highest and lowest firing rates were taken for the comparison. Figure $6 B 1$ shows the mean spatiotemporal maps for the compared high and low rate trials. Theta phase precession had a very similar profile in these two conditions. The mean phase of the action potentials for six sections of the place fields are shown on Figure $6 B 2$. No significant difference was observed between high and low firing rate trials (Watson's two-sample test for circular data; all $p$ values $>0.10$ ). The circular correlations between theta phase and location of the action potentials of each cell were calculated for the two conditions: there was no difference between the strength of the correlation (Fig. $6 \mathrm{B3}$, paired $t$ test; $t=0.79 ; p=0.43$ ). Thus, the spatial temporal code appears to be maintained despite the significant modulations of firing rate associated with the trial type or the previous location of the animal.

The reconstruction of the animal's position from cell activity also suggested that the spatial map made by these cells was unaltered by the type of trials during the task. Reconstructions were performed only when there were at least three spikes per $200 \mathrm{~ms}$ time window and each theta cycle was divided into five bands. The median of the reconstruction error when the rats were running in the MCAZ was $\sim 7 \mathrm{~cm}$ (Fig. $6 \mathrm{C}$ ). In one condition (Same), the position of the rat on each trial was reconstructed using a model built on the other trials of the same type. In the other condition (Diff), each trial was reconstructed using the trials of the other type but with the exception of one randomly selected trial. We found that the type of trials performed by the rat did not affect the quality of the spatial reconstruction in the MCAZ (Fig. 

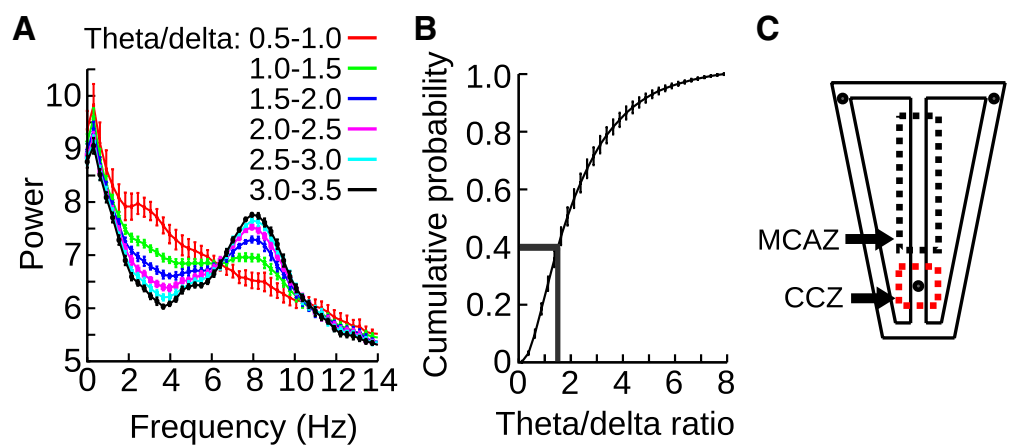

D

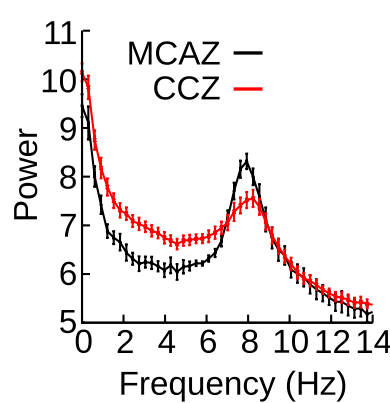

E
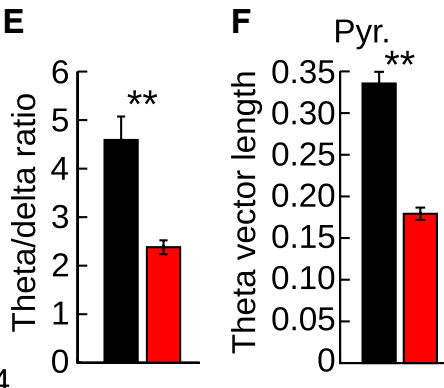

G
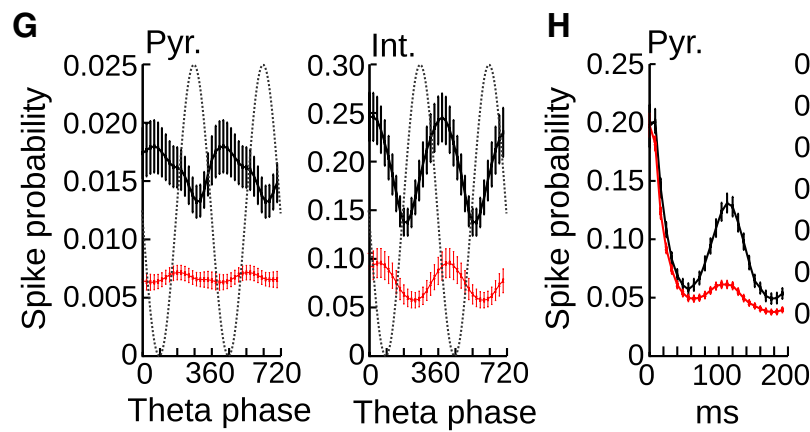

Figure 5. Different network states characterized periods in the CCZ and the MCAZ. A, Power spectra performed on $500 \mathrm{~ms}$ epochs with different theta/delta ratios. A clear theta peak is observed for epochs with a theta/delta ratio larger than 1.5. $\boldsymbol{B}$, Distribution of theta/delta ratios during the recording sessions. Approximately $60 \%$ of the recording sessions were considered as theta epochs when using a theta/delta ratio of 1.5 as detection threshold. $\boldsymbol{C}$, Representation of the maze, the conditional cue zone (red dotted rectangle), and the middle central arm zone (black dotted rectangle). D, Power spectra for the time periods in which the rat was in the $C C Z$ or the MCAZ. E, Mean theta/delta ratio when the rat was in the CCZ or the MCAZ.F, Mean theta vector length for the spikes of pyramidal cells (Pyr.) and interneurons (Int.) when the rat was in the CCZ or the MCAZ. G, Probability of firing at different theta phase for pyramidal cells and interneurons in the CCZ and the MCAZ. $\boldsymbol{H}$, Spike-time autocorrelation for pyramidal cells and interneurons in the $C C Z$ and the MCAZ. ${ }^{*} p<0.01$.

$6 D, E)$ : Predicting the animal's position using a model based on trials of the same or different type led to a similar reconstruction error (Wilcoxon rank sum test, $n=1608$, Trial-Type: $p>0.5$, Previous-Location: $p>0.5$ ). Paired $t$-tests on the median of reconstruction error for the Same and Diff conditions of each recording session revealed no significant difference (Fig. $6 E, p>$ $0.50)$. Simulations in which the location of the firing fields in the MCAZ were shifted randomly along the longitudinal axis of the zone indicated that a change in the firing field location in $10 \%$ of the cells was sufficient to cause a significant increase in reconstruction error (Fig. $6 F$, paired $t$ test on median of recording sessions, comparing no firing field shift with field shift in $10 \%$ of the cells, $p<0.015$ ). Taken together, these findings indicate that the spatial representation of the central arm of the maze was stable across the different conditions.
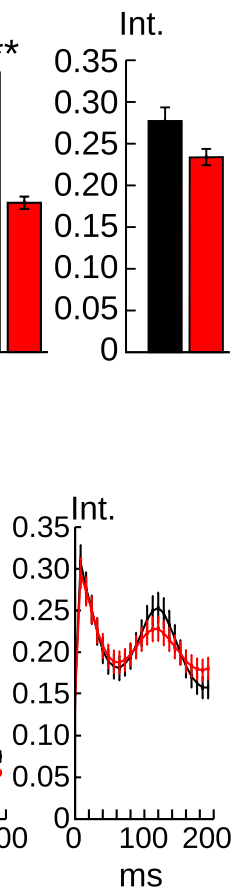

Prediction of trial type, previous

location, and current position of the rat on correct and error trials

Using the firing rate of a group of pyramidal cells, it was possible to predict both the trial type and the previous location of the animal on a single-trial basis. On correct trials, accurate prediction for both the trial type and the previous location occurred on $73.02 \%$ of the trials ( 341 out of 467 trials, Fig. 7A). This was significantly better than chance levels (chance $=25 \%, \chi^{2}=$ 574.31; $\left.p<10^{-16}\right)$. The prediction accuracy for individual recording sessions ranged from 52.38 to $88.23 \%$ and was significantly different from chance levels in all instances (all $p$ values < 0.0001 ). The accuracy of the prediction for the trial type or the previous location alone were higher $(83.72$ and $81.80 \%$, respectively) than the combined prediction. This was significantly above chance levels (chance $=50 \%, \chi^{2}$ trial type $=$ 212.47; $p<10^{-16}, \chi^{2}$ previous location $\left.=188.88 ; p<10^{-16}\right)$.

We then investigated whether the accuracy of the representations for the trial type and previous location covaried during the task. We analyzed the prediction accuracy for a first factor when the prediction of the second factor was correct or incorrect. We found that the likelihood of predicting one factor correctly was strongly linked to the successful prediction of the second factor (Fig. 7B). On correct trials in which the previous location was predicted successfully, the trial type could also be predicted correctly on $89.27 \%$ of the trials. When the previous location was not predicted successfully, then the prediction accuracy for the trial type dropped to $58.82 \%$, which was significantly lower $\left(\chi^{2}=4.30 ; p<0.05\right)$. The prediction accuracy of the previous location also depended on the accuracy of the trial type prediction (Fig. $7 B, p<0.05$ ). This indicated that the quality of the representation of both factors covaried on correct trials.

We tested whether the trial type and the previous location of the animal could be predicted on error trials. On these trials, the animal received the conditional cue but visited the unbaited goal arm. Therefore, the influence of the conditional cue and that of the future location of the animal could potentially be dissociated. Moreover, the animals alternated on these trials $85 \%$ of the time, so they were apparently guided by the location they had visited on the previous trial. Accurate prediction for both the trial type and the previous location occurred on $27.06 \%$ of the trials (Fig. 7C). This was not significantly better than chance levels ( chance $=25 \%, \chi^{2}=0.19$; $p=0.66)$. The accuracy of the prediction for the trial type or the previous location alone was also not significantly above chance levels $\left(\right.$ chance $=50 \%, \chi^{2}$ trial type $=3.4 ; p=0.07, \chi^{2}$ 
A
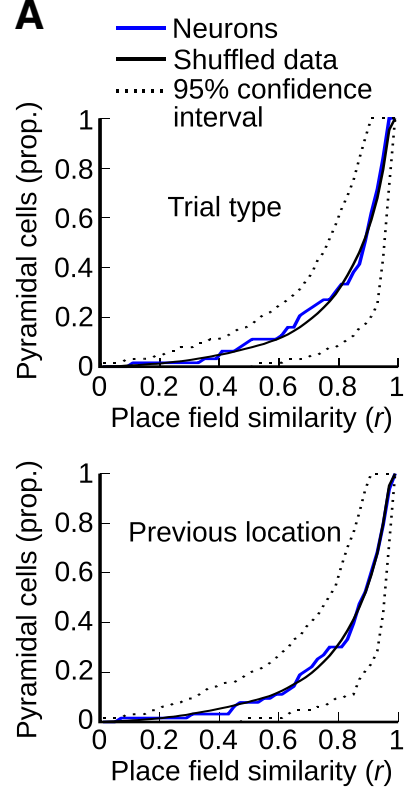

C
B1

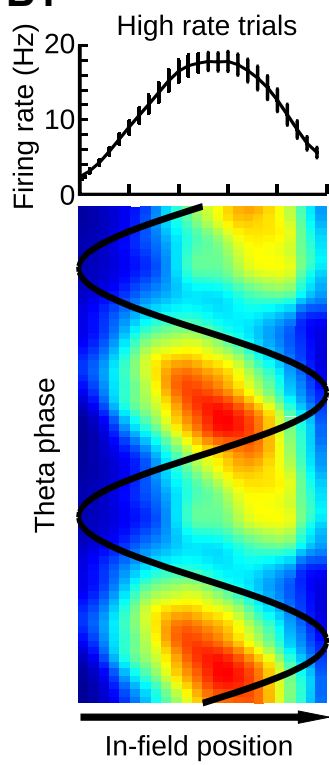

D

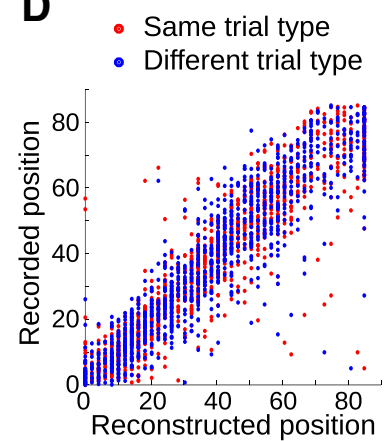

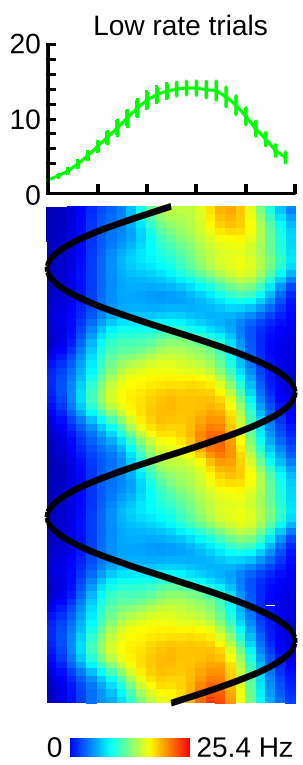

E

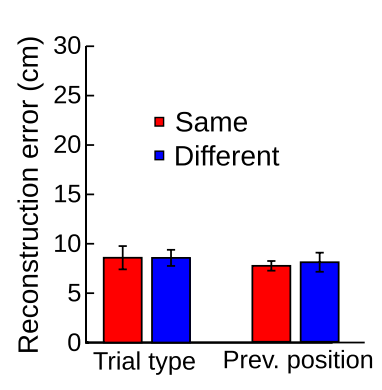

B2
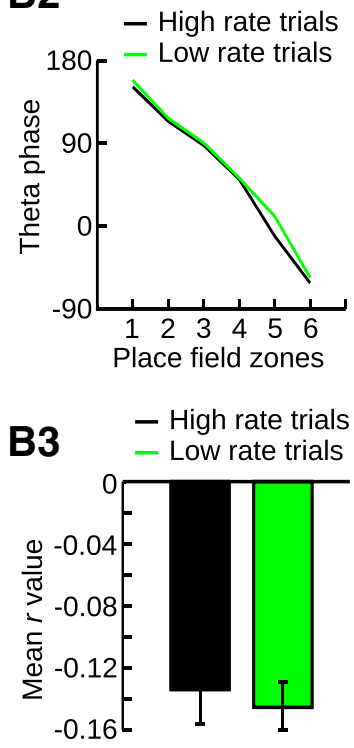

F

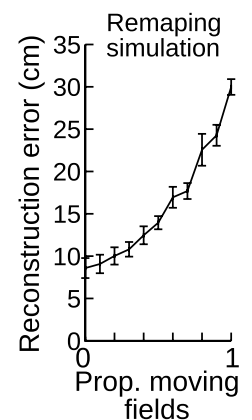

Figure 6. Effect of trial type and previous location on spatial coding in the MCAZ. A, Cumulative distribution of the place field similarity as expressed by the correlation coefficients between the place rate maps of the MCAZ for the two types of trials (top) or the different previous locations (bottom). The distributions were not significantly different from these obtained after shuffling the trial identity. $\boldsymbol{B}$, Place-theta phase relationship for cells showing a significant effect of Trial-Type, Previous-Location, or an interaction of these two factors. When significant interactions were detected, conditions with the highest and lowest firing rate were considered. B1, Top, Mean linear firing rate maps within the place field for conditions associated with high firing rate (high rate trials) and low rate (low rate trials). Bottom, Mean place-phase maps showing theta phase precession. Theta phase precession was observed in both conditions. B2, Mean phase of the spikes for six equal sections of the place fields for different conditions. B3, Mean circular-linear correlation between theta phase and location for spikes during trials with high and low firing rate. $\boldsymbol{C}$, Example of reconstruction of the position of the rat on two different trials. $\boldsymbol{D}$, Reconstruction of the position in the MCAZ from one recording session. The predicted position was strongly correlated to the recorded location of the animal. This relationship was similar for reconstruction made with trials of the same or different type. E, Effect of the trial type or the previous location on the position reconstruction error in the MCAZ. The median was obtained from each recording session and the mean across the sessions was calculated. There was no difference between reconstructions made with trials of the same or different trial types or previous location. $\boldsymbol{F}$, Simulation performed in which the firing fields of different proportions of place cells were randomly moved within the MCAZ. Reconstruction error significantly increased when only $10 \%$ of the firing fields moved $(p<0.015$ ), suggesting that the reconstruction method would be sensitive to a displacement of few firing fields across the different task conditions.

previous location $=0.58 ; p=0.45)$. These results suggest that the representations of the trial type and the previous location of the animal were altered on error trials. Neither the conditional cue, nor the choice behavior of the animal could be predicted on error trials.

Next, we investigated whether the spatial map per se was altered on error trials. The quality of the spatial map during correct and error trials was compared by predicting the location of the animal while the rat was in the MCAZ. We first used correct trials to predict the position of the animal on error trials. The number of correct trials used in the model was equal to the number of correct trials minus the number of error trials. Then we used the same number of correct trials, selected randomly, to predict the position of the animal on correct trials. This last procedure was repeated 500 times to estimate the quality of the prediction on correct trials. We compared the distributions of reconstruction errors on correct and error trials and found that there was a significant tendency for larger error in the reconstruction during error trials (Kolmogorov-Smirnov; $D=0.13$; $p<$ 0.01). The reconstruction error for correct and error trials was then investigated further for time windows with different numbers of spikes (Fig. 7D). The position reconstruction was better during correct trials when more than 10 spikes were recorded during the reconstruction time windows ( 10 to 18 spikes: $D=0.09 ; p<0.01,18$ to 26 spikes: $D=0.13$; $p<0.01)$. This demonstrates that the spatial map made by a population of place cells is less accurate, or altered, during error trials.

Trial-related coding mechanisms during the rewarded alternation task without delays

The results obtained during the conditional discrimination task indicate that hippocampal cells encode trial-related information 

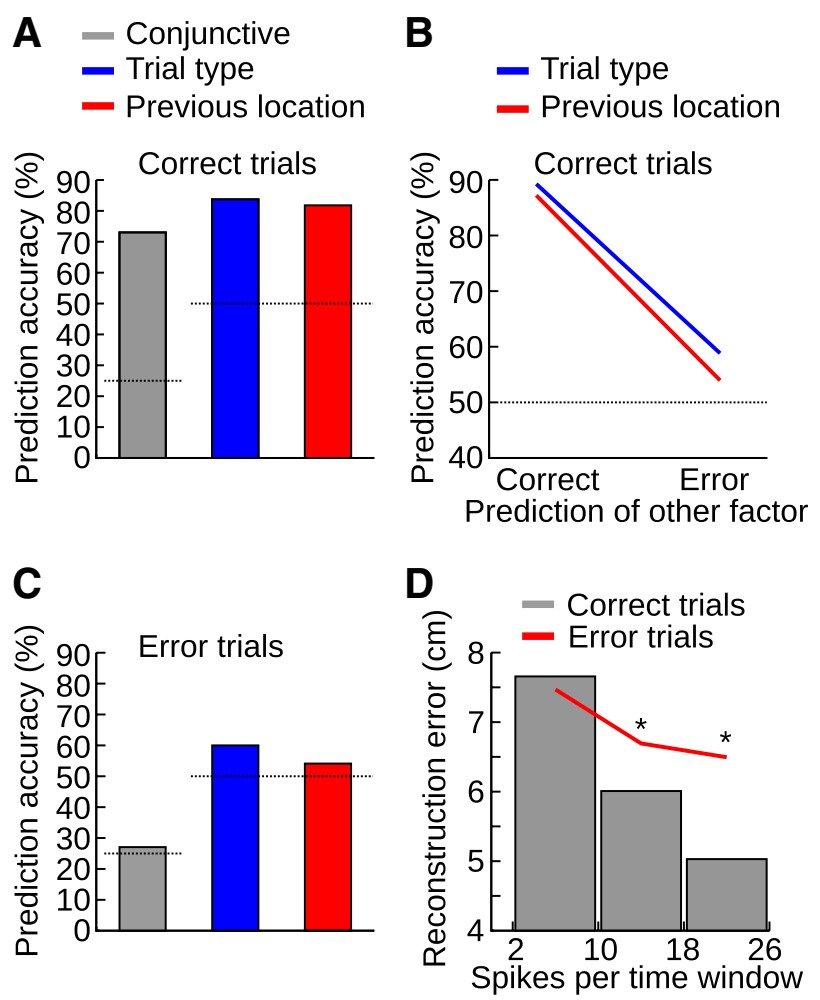

Figure 7. Prediction of the trial type, previous location, and current position based on cell activity during correct and error trials. $\boldsymbol{A}$, Prediction accuracy for the trial type and previous location using the firing rate of a group of cells during correct trials. Prediction of conjunctive information means that both the trial type and the previous location were predicted successfully. Chance levels are indicated with dotted lines. The prediction accuracy was significantly above chance levels. $\boldsymbol{B}$, The prediction accuracy for the two factors on correct trials covaried. The prediction accuracy for one factor was above chance level only on trials where the other factor could be predicted successfully. $\boldsymbol{C}$, The prediction for the trial type and the previous location on the error trials. Prediction accuracy on error trials was not significantly different from chance levels. $\boldsymbol{D}$, Reconstruction of the animal position on correct and error trials for reconstruction time windows with different number of spikes. The spatial reconstruction was significantly impaired on error trials only when $>10$ spikes were recorded during the reconstruction time window. ${ }^{*} p<0.01$.

mainly via changes in firing rate, hence rate remapping underlies their task-related coding. We next tested whether rate remapping also applies to other memory tasks in which the future choice of the animal is guided by its previous location. We recorded the activity of pyramidal cells in rats performing the continuous version of the rewarded alternation task without delays (Fig. 8A). The rats were implanted with 16 tetrodes before being trained on the task. We recorded the activity of hippocampal pyramidal cells during six recording sessions. The mean performance during these sessions was $92.72 \%$ correct responses, with all recording sessions above $80 \%$ (Fig. 8 B). A total of 971 pyramidal cells were recorded on the maze.

As expected, pyramidal cells changed their firing rate on the central arm of the maze depending on the ongoing trial type (left-to-right or right-to-left) (Wood et al., 2000; Lee et al., 2006). A total of 141 cells fired at least 300 spikes in the central arm zone. For each cell, an ANCOVA was performed to establish whether the factors Trial-Type and longitudinal Y-Position had an effect on the firing rate of the cell, while controlling for the potential effects of speed and the lateral position of the animal. Fifty-nine cells (41.84\%) showed either a significant effect of Trial-Type or a Trial-Type versus Y-Position interaction (Fig. 8C). The mean $F$ value for the effect of Trial-Type in the real data was larger than what could be expected by chance (Fig. $8 D$, mean $F$ value: 17.58 , smallest mean $F$ value in shuffled samples with a probability $<0.01: 3.63)$. Thus, a significant proportion of cells changed their firing rate during the rewarded alternation task depending on the type of trials (left-to-right or right-to-left).

As illustrated with the cells presented in Figure $8 C$, most cells had a field at a similar location on the two types of trials. For each cell, a correlation was performed between the place rate maps obtained from trials of different types. The mean correlation coefficient between the place rate maps was $0.87 \pm 0.01$ (Fig. $8 E$ ). The mean correlation coefficient was not significantly lower than what could be expected by chance (mean $r$ value in shuffled samples with a probability $<0.05: 0.80$ ). Thus, the majority of pyramidal cells fired at a similar location during both types of trials. The algorithm to reconstruct the position of the rat from cell activity was also applied to data from the rewarded alternation task (Fig. $8 F$ ). The median of the reconstruction error when the rat was running in the central arm zone was 5.71 and $5.80 \mathrm{~cm}$ when predicting the spatial position of the rat using a model based on trials of the same or different type, respectively (Kolmogorov-Smirnov test, $n=3333, p=0.24$ ). Paired $t$-tests on the median of the reconstruction error for the Same and Diff conditions of each recording sessions revealed no significant difference (Fig. $8 G, p>0.50$ ). Simulations in which the location of the firing fields was shifted along the longitudinal axis of the central arm zone showed that global remapping would have severely affected reconstruction error (Fig. $8 H$ ) since even the shuffling of place fields in $10 \%$ of the cells yielded significant changes in the prediction distance error $(p<0.01)$.These findings indicate that the spatial representation of the central arm of the maze was stable during the continuous version of the rewarded alternation task without delays and that memory-related coding during this task is expressed mainly via rate changes of stable place fields.

\section{Discussion}

This study shows that the firing of hippocampal pyramidal cells can encode several trial-related features or even the combination of these features during a conditional discrimination task. These trial-related features were encoded through firing rate modulations within the place field without significant changes in place field location per se, demonstrating the rate remapping of place fields. Trial-related rate changes were present during two distinct phases of the conditional discrimination task, which were characterized by a different influence of theta oscillations on the activity of hippocampal neurons. This rate modulation accurately predicted the behavior of the animal on correct but not error trials, suggesting that this form of rate-coding may contribute to the animal's behavioral performance. In addition, we show that during the continuous version of the rewarded alternation task without delays, conditional firing also occurs via rate-remapping of spatially stable place cells.

\section{Trial-related changes in the firing rate of place cells}

We were able to show that two independent trial-related features can be encoded by hippocampal cells, and some cells can even encode the combinations of these features. In the conditional discrimination task, nonspatial conditional cues (different food flavors) signaled to the rat which goal arm of the maze it had to visit next, and these conditional cues were randomly assigned across consecutive trails. We have found place cells that encoded the current trial type (associated with the conditional cue and the future choice of the animal), the past location (i.e., past choice of the animal), or the combination of both. Hence, cells could encode multiple trial-related features. Our results also show that firing rate changes associated with the future choice of the rat can 
be updated on a trial-to-trial basis, which is a shorter time scale than in previous studies in which firing responses were compared between long-duration trial blocks only (Ferbinteanu and Shapiro, 2003; Ainge et al., 2007a; Kennedy and Shapiro, 2009; but see Ainge et al., 2012).

In the conditional discrimination task, trial-related firing was observed when the rat paused at the start of the central arm, where it was presented with the conditional cue. The speed of the animal in that zone was much lower and the power of theta activity was reduced. Interestingly, interneurons maintained theta modulated firing, whereas pyramidal cells lose much of their theta modulation. We were able to differentiate cells that encoded the current or the past trial type or even combinations of these during that state. These cells are unlikely to exhibit theta phase precession in this condition, due to their very weak theta phase modulation and the suppressed theta modulation in their spike-time autocorrelations. These responses were different from conditional responses observed while an animal was running on a wheel in delay periods; there cells were theta modulated and exhibited theta phase precession (Pastalkova et al., 2008). Some of our cells exhibited trial type versus time interactions near the food well, suggesting that, depending on the trial types, they fired at different relative time periods near the food well (Fig. 3 ). The firing of these cells was reminiscent of "time cells" that exhibit timedependent sequences in delay periods (MacDonald et al., 2011). Overall, the network patterns observed near the conditional cue food well could represent an intermediate state in which trialdependent firing remains in the presence of reduced theta oscillatory activity, whereas the theta phase of cells no longer signals spatial locations.

\section{Possible contributions of spatial and trial-related information to the animal's performance}

Using the firing rate of pyramidal cells, it was possible to predict both the current and previous trial types, i.e., the future response of the animal and its previous location on the maze. On correct trials, the accuracy of this prediction was above $70 \%$, which is much higher than chance levels (25\%). Moreover, the ability to predict the previous location of the animal and the conditional cue were linked on a trial-to-trial basis; when the activity of the hippocampal cell population accurately predicted the previous location of the an-
A
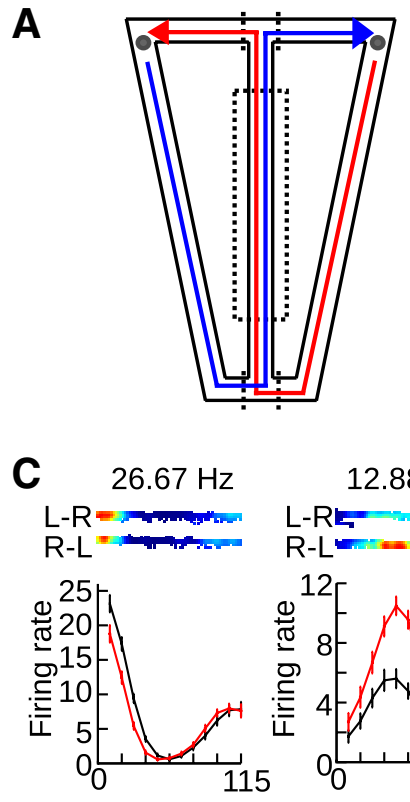

Position $(\mathrm{cm})$
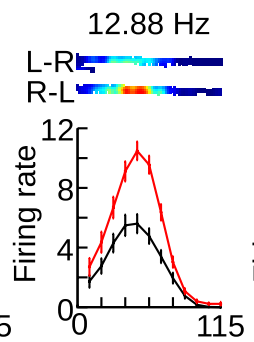

Position (cm)

\section{D}
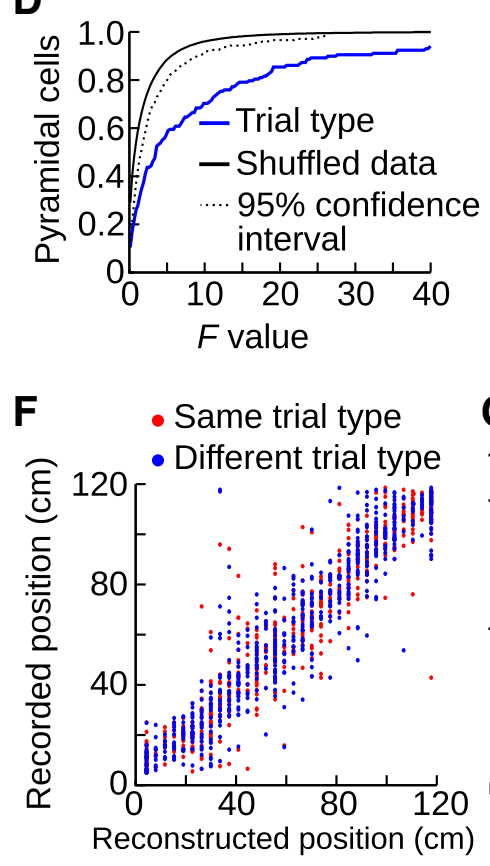

B

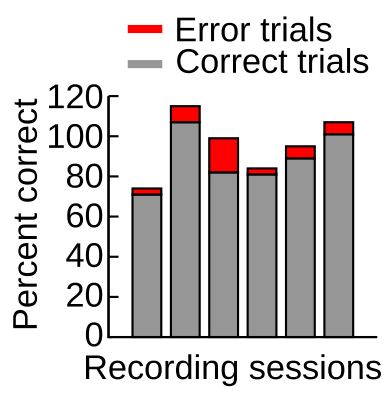

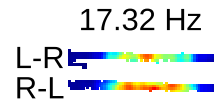

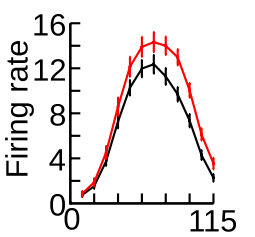

Position (cm)
E
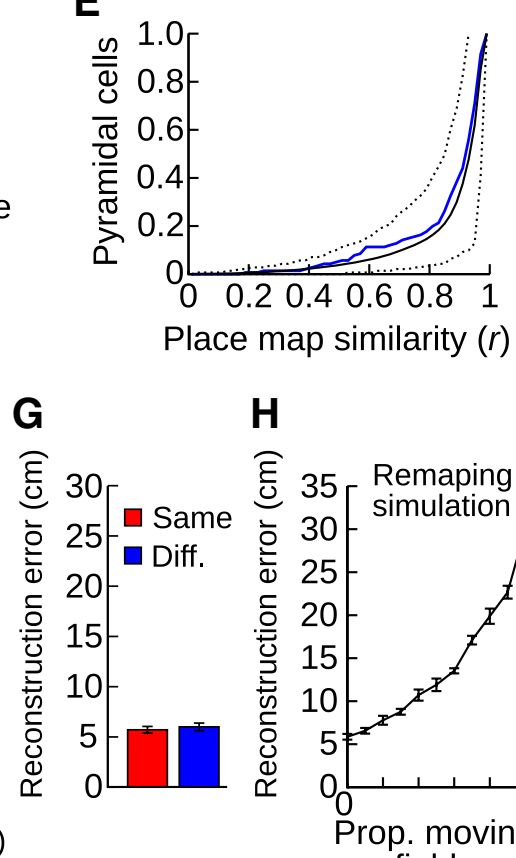

Figure 8. Trial-related changes in firing rate during rewarded alternation without delays. $\boldsymbol{A}$, Schematic of the continuous version of the rewarded alternation task without delays on the modified T-maze. The location of each inverted guillotine door used in the experiment is indicated by a dotted line. Gray circles represent the food wells. The dotted rectangle corresponds to the zone used for the analysis on the central arm. The two colored arrows indicate the two trial types (left-to-right in blue and right-to-left in red). $\boldsymbol{B}$, Number of trials and performance during the recording sessions used for electrophysiological analysis. Each band of the graph represents a recording session. C, Examples of four pyramidal cells showing significant changes in the firing rate depending on the trial type. The density plots show place rate maps of the cells on the central arm for the two trial types ( $L-R$, left-to-right; $R-L$, right-to-left). The number above each density plot is the peak firing rate of the cell. The graphs show the mean firing rate ( \pm SEM) of the same cells in a linear representation of the central arm. $D$, Cumulative distribution of the $F$ values obtained for the effect of trial type during recording sessions and that obtained after shuffling the identity of the trials. $\boldsymbol{E}$, Cumulative distribution of the place field similarity, expressed as the correlation coefficients between the place rate maps of the central arm of the maze for the two types of trials. The distribution was not significantly different from that obtained after shuffling the trial identity. $\boldsymbol{F}$, Reconstruction of the position on the central arm of the maze from one recording session. The predicted position was strongly correlated to the recorded location of the rat. $\mathbf{G}$, Effect of the trial type (left-to-right and right-to-left) on the position reconstruction error. The median was obtained from each recording session and the mean across the sessions was calculated. There was no difference between reconstructions made with trials of the same or different (Diff.) trial types. $\boldsymbol{H}$, Simulation in which the firing fields of different proportions of place cells were randomly moved within the central arm zone. 
imal, the prediction of the conditional stimulus was more likely to be accurately predicted as well. This finding suggests that these two variables are interrelated.

On error trials, the prediction of the conditional cue was at chance levels. This shows that on error trials the activity of hippocampal neurons did not encode correctly the trial type determined by the conditional cue. Neither had it predicted the wrong choice of the animal, as, in that case, hippocampal activity should have signaled the opposite conditional cue. Hippocampal activity did not predict the previous location of the animal on error trials either. Therefore, unlike on correct trails, hippocampal neurons failed to signal trial-related information during error trials. Together, these results suggest that correct encoding of trial-related information could contribute to the behavioral performance of the animal.

A second contribution of the hippocampus to the conditional memory task could be to provide a spatial map of the animal on the maze. On error trials we found that the spatial reference frame of place cells was impaired: the spatial map made by hippocampal cells during error trials was not as accurate as that on correct trials. Therefore, our results point toward a dual-role for the hippocampus in our task: it may simultaneously provide allocentric spatial information as well as task-contingent information to support the animal's behavior. These functions could be supported by distinct codes for the trial-related and spatial information, both supporting the behavioral performance of the animal.

\section{Interactions between spatial and trial-related coding in the hippocampus}

There are at least two possible scenarios explaining how trialrelated information interacts with spatial coding in the hippocampus. In one scenario, some place cells might be active only during some trials or the location of some firing fields could change across trials. This partial remapping should lead to the activation of different spatial maps during the different trial types as suggested by some studies (Ferbinteanu and Shapiro, 2003; Kennedy and Shapiro, 2009). Alternatively, other studies put forward that maps may remain the same in different trial conditions but the in-field rate of place cells may change depending on trial type (Lipton et al., 2007; Ainge et al., 2012). Indeed, such a rate remapping has been observed in conditions in which environmental features have been altered (Leutgeb et al., 2005). Here, we quantitatively examined whether task-related firing observed in mazes is due to global, partial, or rate remapping. We found that, in two different memory tasks (i.e., conditional discrimination task and rewarded alternation task without delays), trial-related information was encoded by firing rate changes inside the place fields without affecting the location of the place fields themselves. In fact, place maps measured on the opposite trial type could still be used to accurately predict the current location of the animal. In addition, we observed no change in the theta-phase precession profile of pyramidal cells in conditions associated with the encoding of different trial-related features. These results demonstrate that both place and trial-related information can be encoded in the CA1 region using a combined temporal-rate code in which the firing theta phase of the cells reflected the animal's position, whereas the firing rate varied on a trial-to-trial basis to reflect contextual information. Interestingly, it was recently suggested that rate remapping could be the mechanism by which pyramidal cells signal the previous location of the animal during a plusmaze task in which the animal has to run from one of a pair of opposite start arms to a goal arm (Ferbinteanu et al., 2011). Also, given the recent differences observed between the firing of CA1 and CA3 pyramidal cells during memory tasks (Bahar et al., 2011), future experiments will be needed to assess whether similar coding mechanisms operate in the different hippocampal subregions.

We have been able to demonstrate that the firing rate of hippocampal neurons can encode not only single trail-related features but also a combination of these features. These conjunctive place cells changed their firing rate to reflect both the previous location of the animal and the future location associated with the conditional cue. This suggests that, once large populations of cells are considered, firing rate modulation can encode combinations of complex, trial-related features by forming distinct cell assemblies. Moreover, sequentially firing place cells that encode conjunctive information could potentially encode event sequences, which would allow the hippocampus to contribute to a representation of associations between different dimensions of an episode (Eichenbaum and Cohen, 2004; Foster and Wilson, 2006; Pastalkova et al., 2008; MacDonald et al., 2011). This type of conjunctive coding by hippocampal neurons could potentially allow the animal to learn conditional rules that depend on a combination of several conditional stimuli.

\section{References}

Ainge JA, Tamosiunaite M, Woergoetter F, Dudchenko PA (2007a) Hippocampal CA1 place cells encode intended destination on a maze with multiple choice points. J Neurosci 27:9769-9779.

Ainge JA, van der Meer MA, Langston RF, Wood ER (2007b) Exploring the role of context-dependent hippocampal activity in spatial alternation behavior. Hippocampus 17:988-1002.

Ainge JA, Tamosiunaite M, Wörgötter F, Dudchenko PA (2012) Hippocampal place cells encode intended destination, and not a discriminative stimulus, in a conditional T-maze task. Hippocampus 22:534-543.

Bahar AS, Shirvalkar PR, Shapiro ML (2011) Memory-guided learning: CA1 and CA3 neuronal ensembles differentially encode the commonalities and differences between situations. J Neurosci 31:12270-12281.

Bower MR, Euston DR, McNaughton BL (2005) Sequential-contextdependent hippocampal activity is not necessary to learn sequences with repeated elements. J Neurosci 25:1313-1323.

Csicsvari J, Hirase H, Czurkó A, Mamiya A, Buzsáki G (1999) Oscillatory coupling of hippocampal pyramidal cells and interneurons in the behaving Rat. J Neurosci 19:274-287.

Eichenbaum H, Cohen NJ (2004) From conditioning to conscious recollection, Oxford UP.

Ferbinteanu J, Shapiro ML (2003) Prospective and retrospective memory coding in the hippocampus. Neuron 40:1227-1239.

Ferbinteanu J, Shirvalkar P, Shapiro ML (2011) Memory modulates journeydependent coding in the rat hippocampus. J Neurosci 31:9135-9146.

Foster DJ, Wilson MA (2006) Reverse replay of behavioural sequences in hippocampal place cells during the awake state. Nature 440:680-683.

Frank LM, Brown EN, Wilson M (2000) Trajectory encoding in the hippocampus and entorhinal cortex. Neuron 27:169-178.

Huxter J, Burgess N, O'Keefe J (2003) Independent rate and temporal coding in hippocampal pyramidal cells. Nature 425:828-832.

Jeffery KJ, Anderson MI (2003) Dissociation of the geometric and contextual influences on place cells. Hippocampus 13:868-872.

Jensen O, Lisman JE (2000) Position reconstruction from an ensemble of hippocampal place cells: contribution of theta phase coding. J Neurophysiol 83:2602-2609.

Kennedy PJ, Shapiro ML (2009) Motivational states activate distinct hippocampal representations to guide goal-directed behaviors. Proc Natl Acad Sci U S A 106:10805-10810.

Lee I, Griffin AL, Zilli EA, Eichenbaum H, Hasselmo ME (2006) Gradual translocation of spatial correlates of neuronal firing in the hippocampus toward prospective reward locations. Neuron 51:639-650.

Leutgeb S, Leutgeb JK, Barnes CA, Moser EI, McNaughton BL, Moser MB (2005) Independent codes for spatial and episodic memory in hippocampal neuronal ensembles. Science 309:619-623.

Lipton PA, White JA, Eichenbaum H (2007) Disambiguation of overlap- 
ping experiences by neurons in the medial entorhinal cortex. J Neurosci 27:5787-5795.

MacDonald CJ, Lepage KQ, Eden UT, Eichenbaum H (2011) Hippocampal "time cells" bridge the gap in memory for discontiguous events. Neuron 71:737-749.

O'Keefe J, Dostrovsky J (1971) The hippocampus as a spatial map. Preliminary evidence from unit activity in the freely-moving rat. Brain Res 34:171-175.

O’Keefe J, Recce ML (1993) Phase relationship between hippocampal place units and the EEG theta rhythm. Hippocampus 3:317-330.

Pastalkova E, Itskov V, Amarasingham A, Buzsáki G (2008) Internally generated cell assembly sequences in the rat hippocampus. Science 321:1322-1327.

Press WH, Teukolsky SA, Vetterling WT, Flannery BP (2007) Numerical recipes 3rd edition: the art of scientific computing. Cambridge: Cambridge UP.

Shapiro ML, Ferbinteanu J (2006) Relative spike timing in pairs of hippocampal neurons distinguishes the beginning and end of journeys. Proc Natl Acad Sci U S A 103:4287-4292.

Wood ER, Dudchenko PA, Eichenbaum H (1999) The global record of memory in hippocampal neuronal activity. Nature 397:613-616.

Wood ER, Dudchenko PA, Robitsek RJ, Eichenbaum H (2000) Hippocampal neurons encode information about different types of memory episodes occurring in the same location. Neuron 27:623-633.

Zhang K, Ginzburg I, McNaughton BL, Sejnowski TJ (1998) Interpreting neuronal population activity by reconstruction: unified framework with application to hippocampal place cells. J Neurophysiol 79:1017-1044. 